\title{
PROPERTIES OF SOLUTIONS OF AN INFINITE SYSTEM OF ORDINARY LINEAR DIFFERENTIAL EQUATIONS OF THE FIRST ORDER WITH AUXILIARY BOUNDARY CONDITIONS*
}

\author{
BY \\ WILLIAM T. REID \\ 1. Introduction
}

It is the purpose of this paper to consider an infinite system of ordinary linear differential equations of the first order. We write the system considered in the form

$$
\frac{d y_{i}}{d x}=\sum_{\alpha=1}^{\infty} A_{i \alpha}(x) y_{\alpha} \quad(i=1,2, \cdots),
$$

where $y(x) \equiv\left(y_{i}(x)\right)$ is a vector in Hilbert space, i.e., $\sum_{\alpha=1}^{\infty} y_{\alpha}^{2}$ converges and $A(x) \equiv\left(A_{i j}(x)\right)$ is a matrix each of whose elements is a Lebesgue summable function on $X: 0 \leqq x \leqq 1$. Furthermore, the matrix $A(x)$ is limited, in the sense defined by Hilbert, by a Lebesgue summable function $\phi(x)$ on $X$. In $\$ 2$ preliminary definitions are given and the system of notation used throughout the paper is explained.

In $\$ 3$ the properties of matrices of functions which satisfy system (1) and the corresponding adjoint system are considered. The adjoint system is given by

$$
\frac{d z_{i}}{d x}=-\sum_{\alpha=1}^{\infty} A_{\alpha i}(x) z_{\alpha} \quad(i=1,2, \cdots) .
$$

In $\S 4$ we treat a system in which the matrix $A$ of the system (1) is a function of a parameter $\mu$, and sufficient conditions for the solution $y(x ; \mu)$ to be continuous in $(x, \mu)$ are given. The differentiability of the solution with respect to the parameter $\mu$ is also considered.

In $\$ 5$ we set up the system consisting of the equations of (1), together with boundary conditions that involve the value of the solution at two points. The set of boundary conditions that are adjoint to these is determined in a manner similar to that used by Bounitzky $\dagger$ for a finite system of ordinary linear differential equations of the first order.

* Presented to the Society, December 1, 1928, and March 29, 1929; received by the editors in June, 1929.

$\dagger$ E. Bounitzky, Sur la fonction de Green des équations différentielles linéaires ordinaires, Journal de Mathématiques, (6), vol. 5 (1909), pp. 65-125. 
There is treated in $\$ 6$ an infinite system in which each element of the matrix $A$ is a function of a parameter $\mu$ and $A(x ; \mu)$ is limited by a summable function $\phi(x)$ uniformly with respect to $\mu$; furthermore, for each value of $\mu$ and for values of $x$ "almost everywhere" on $X$ the matrix $A(x ; \mu)$ is completely continuous or "vollstetig," as defined by Hilbert.* This system, together with a special class of two-point boundary conditions, is shown to have many properties of a finite system.

Whenever the differential system of $\$ 6$ is incompatible, it is possible to define a Green's matrix for the system. In $\$ 7$ the existence and uniqueness of the Green's matrix is proved. We also state a theorem on defining a Green's matrix for values of the parameter which are characteristic numbers of the system. This theorem is more general than a theorem which has recently been established by W. M. Whyburn $\dagger$ for a finite differential system.

Boundary value problems for a finite system of ordinary linear differential equations of the first order whose coefficients are continuous, have been considered by Birkhoff and Langer $\ddagger$ and by Bliss. $\S$ The results of $\$ \$ 5$ and 6 are an extension to an infinite system of some of the results obtained by Birkhoff and Langer and by Bliss for finite systems. More recently W. M. Whyburn\| has considered a finite system of linear equations whose coefficients are Lebesgue summable functions and has shown that the solution of the differential system is the limit of the solution of the associated algebraic system.

W. L. Hart $\uparrow$ has treated infinite systems of differential equations in four papers. The system considered in the first paper is of a different nature from the system here considered since the variables are defined in a more general

* D. Hilbert, Grundzüge einer allgemeinen Theorie der linearen Integralgleichungen, Berlin, 1912, p. 147.

$\dagger$ W. M. Whyburn, On the Green's function for systems of differential equations, Annals of Mathematics, (2), vol. 28 (1927), pp. 291-300.

‡ G. D. Birkhoff and R. E. Langer, The boundary problems and developments associated with a system of ordinary linear differential equations of the first order, Proceedings of the American Academy of Arts and Sciences, vol. 58 (1923), pp. 51-128.

$\$$ G. A. Bliss, $A$ boundary value problem for a system of ordinary linear differential equations of the first order, these Transactions, vol. 28 (1926), pp. 561-584.

\| W. M. Whyburn, On related difference and differential systems, American Journal of Mathematics, vol. 51 (1929), pp. 265-286.

『 W. L. Hart, Differential equations and implicit functions in infinitely many variables, these Transactions, vol. 18 (1917), pp. 125-160; Linear differential equations in infinitely many variables, American Journal of Mathematics, vol. 39 (1917), pp. 407-424; The Cauchy-Lipschitz method for infinite systems of differential equations, American Journal of Mathematics, vol. 43 (1921), pp. 226-231; Functions of infinitely many variables in Hilbert space, these Transactions, vol. 23 (1922), pp. 30-50. 
space than Hilbert space. In the third paper it is proved by an extension to infinite systems of the notion of Cauchy polygons, and without the assumption of a Lipschitz condition, that the system of equations considered in the first paper has at least one solution satisfying arbitrary initial conditions. In the second paper an infinite linear system, in which the matrix of coefficients is a limited matrix and is analytic in the independent variable, is treated. The results there obtained are special cases of some of the results we obtain in $\$ \S 3$ and 4 . In the fourth paper existence theorems for infinite differential equations in Hilbert space are given. However, system (1) that we consider does not come under Hart's theorem since we suppose that the elements of the matrix of coefficients are only Lebesgue summable functions. On page 408 of Hart's second paper references to earlier literature on infinite differential systems are given. More recently Feenberg* has treated an infinite system of equations in abstract space. Ettlinger $\dagger$ has considered an infinite system of the form (1) in which the elements of the matrix $A(x)$ are summable and in which the matrix $A(x)$ satisfies a condition which we show to be equivalent to the condition that is imposed in this paper. The results of this paper extend those that have been obtained by Ettlinger.

I desire to express my indebtedness to Professor H. J. Ettlinger who suggested to me the topic of this paper and who has assisted in its preparation by many suggestions and criticisms.

\section{Preliminary Definitions and notation}

An infinite set of real numbers $\xi_{1}, \xi_{2}, \cdots$ represents a point or a vector in Hilbert real space provided the sum $\sum_{=1}^{\infty} \xi_{\alpha}^{2}$ converges. If $\xi \equiv\left(\xi_{\alpha}\right)$ and $\eta \equiv\left(\eta_{\alpha}\right)$ are two vectors the dot product of $\xi$ and $\eta$ is the $\operatorname{sum} \sum_{\alpha=1}^{\infty} \xi_{\alpha} \eta_{\alpha}$ and is denoted by $\xi_{\eta}$. The quantity $\left(\sum_{\alpha=1}^{\infty} \xi_{\alpha}^{2}\right)^{1 / 2}$ is the length or modulus of the vector $\xi$ and is denoted by $|\xi|$.

If $M$ is an infinite matrix we denote by $M_{i j}$ the element in the $i$ th row and the $j$ th column of $M$. All matrices considered in this paper, unless otherwise specified, are supposed to be real and to have an infinite number of rows and columns. When we say that a matrix or a vector has a property, which is not essentially a matrix or a vector property, we mean that each element of the matrix or each component of the vector has that property.

We consider in particular matrices that are limited matrices, as defined by Hilbert. $\ddagger$

* For abstract of paper, see Bulletin of the American Mathematical Society, vol. 34 (1928), p. 709.

† For abstract of paper, see Bulletin of the American Mathematical Society, vol. 34 (1928), p. 708.

$\ddagger$ D. Hilbert, loc. cit., p. 125. 
Definition. A matrix $M \equiv\left(M_{i j}\right)$ is said to be limited if there exists a constant $K$ such that for every pair of points $\xi$ and $\eta$ of Hilbert space and for every integer $n$,

$$
\left|\sum_{i=1}^{n} \sum_{j=1}^{n} M_{i j} \xi_{i} \eta_{j}\right| \leqq K\left(\sum_{i=1}^{n} \xi_{i}{ }^{2}\right)^{1 / 2}\left(\sum_{j=1}^{n} \eta_{j}{ }^{2}\right)^{1 / 2} .
$$

When such a constant exists we say that the matrix $M$ is limited by the constant $K$. Properties of limited matrices have been considered by Hellinger and Toeplitz.* We will use the notation "H.T., page ——" to refer to a page in this article.

If $M \equiv\left(M_{i j}\right)$ and $N \equiv\left(N_{i j}\right)$ are two limited matrices, the sum matrix $M+N$ is given by $M+N \equiv\left(M_{i j}+N_{i j}\right)$ and is also a limited matrix. If $k$ is a scalar and $M$ is a matrix, then $k M \equiv\left(k M_{i j}\right)$. In particular, the zero matrix is the matrix each of whose elements is zero, and $E$ is the unit matrix $E \equiv\left(E_{i j}\right)$, where $E_{i j}=0$ if $i \neq j$ and $E_{i i}=1$. If $M$ is a matrix the adjoint matrix $\bar{M}$ is defined by $\bar{M} \equiv\left(\bar{M}_{i j}\right)$, where $\bar{M}_{i j}=M_{j i}$. We denote the vector $v \equiv\left(v_{i}\right)$, where $v_{i}=M_{i \alpha} \xi_{\alpha}$, by $M \xi$. In this expression $\alpha$ is an umbral subscript. That is, $M_{i \alpha} \xi_{\alpha}=M_{i 1} \xi_{1}+M_{i 2} \xi_{2}+\ldots$. Whenever it is possible to do so without causing confusion, umbral subscripts will be used. Similarly, if $\xi$ is any vector in Hilbert space the vector $u \equiv\left(u_{i}\right)$, where $u_{i}=M_{\alpha i} \xi_{\alpha}$, will be denoted by $\xi M$. If $M$ and $N$ are limited matrices, then the product $M N$ is given by $M N \equiv\left(M_{i \alpha} N_{\alpha j}\right)$. Since it is shown by Hellinger and Toeplitz that each row and each column of a limited matrix is a vector in Hilbert space, the matrix $M N$ is well-defined and is also a limited matrix. In general $M N \neq N M$.

Let $v^{(1)}, v^{(2)}, \cdots$ be an infinite sequence of vectors, where $v^{(n)} \equiv\left(v_{i}^{(n)}\right)$. If there exists a vector $v \equiv\left(v_{i}\right)$ such that $\lim _{n \rightarrow \infty} v_{i}{ }^{(n)}=v_{i}(i=1,2, \cdots)$ we will say that the sequence of vectors converges to the vector $v$ and write $\lim _{n \rightarrow \infty} v^{(n)}=v$. If there exists a vector $v$ so that $\lim _{n \rightarrow \infty}\left|v^{(n)}-v\right|=0$ we will say that the sequence of vectors converges strongly $\dagger$ to the limit vector $v$ and write $\operatorname{LIM}_{n \rightarrow \infty} v^{(n)}=v$.

Let $v(x)$ for each value of the variable $x$ on $a \leqq x \leqq b$ denote a vector in Hilbert space. If $x_{0}$ is a point on the interval $(a, b)$ and for every sequence of points $x_{1}, x_{2}, \cdots$ on this interval having $x_{0}$ as a sequential limit point we have $\operatorname{LIM}_{n \rightarrow \infty} v\left(x_{n}\right)=v\left(x_{0}\right)$, we say that the vector $v(x)$ is strongly continuous at $x=x_{0}$. If $v(x)$ is strongly continuous at every point on $(a, b)$, then $v(x)$ is said to be strongly continuous on this interval.

* E. Hellinger and O. Toeplitz, Grundlagen für eine Theorie der unendlichen Matrizen, Mathematische Annalen, vol. 69 (1910), pp. 289-330.

$\dagger$ The idea of strong convergence was introduced by Hilbert. See Hilbert, loc. cit., p. 127. See also F. Riesz, Les Systèmes d'Équations Linéaires à une Infinité d'Inconnues, Paris, 1913, p. 59. 
Vitali* has proved the following theorem which will be used frequently in this paper.

TheOREM 2.1. Let $f_{1}(x), f_{2}(x), \cdots$ be a sequence of Lebesgue summable functions of the bounded point set $X$ and such that $\lim _{n \rightarrow \infty} f_{n}(x)=f(x)$ on $X_{0} . \dagger$ If the absolute continuity of the integrals $\int f_{n}(x) d x$ is uniform on the set $X$, then $f(x)$ is a Lebesgue summable function on $X$ and

$$
\lim _{n \rightarrow \infty} \int_{X} f_{n}(x) d x=\int_{X} f(x) d x .
$$

We now state without proof the following corollaries to this theorem:

CoROLlary 1. Under the above hypotheses $\int_{T} f_{n}(x) d x$ converges to $\int_{T} f(x) d x$ uniformly for all measurable subsets $T$ of $X$. That is, for every $\epsilon>0$ there is a positive $N(\epsilon)$ such that if $n>N(\epsilon)$ then for every measurable subset $T$ of $X$ we have

$$
\left|\int_{T} f_{n}(x) d x-\int_{T} f(x) d x\right| \leqq \epsilon .
$$

CoRollary 2. If $\sum_{\alpha=1}^{\infty} A_{\alpha}(x)$ is a series of absolutely continuous functions which converges on the interval $X: a \leqq x \leqq b$ and the series

$$
\sum_{\alpha=1}^{\infty} \frac{d}{d x} A_{\alpha}(x)
$$

converges on $X_{0}$, and furthermore the absolute continuity of the integrals

$$
\int \sum_{\alpha=1}^{n} \frac{d}{d x} A_{\alpha}(x) d x
$$

is uniform with respect to $n$, then $\sum_{\alpha=1}^{\infty} A_{\alpha}(x)$ is an absolutely continuous function on $X$ and

$$
\frac{d}{d x}\left[\sum_{\alpha=1}^{\infty} A_{\alpha}(x)\right]=\sum_{\alpha=1}^{\infty} \frac{d}{d x} A_{\alpha}(x) \text { on } X_{0} .
$$

Ettlinger $\ddagger$ has recently stated a theorem on the continuity of a function

* M. Vitali, Sull'integrazione per serie, Rendiconti del Circolo Matematico di Palermo, vol. 23 (1907), pp. 137-155.

$\dagger X_{0}$ is used to denote "almost everywhere" on $X$, or $X$ minus a null set. It is used in the generic sense and the excepted null set may vary.

$\ddagger$ H. J. Ettlinger, On continuity in several variables, Bulletin of the American Mathematical Society, vol. 33 (1927), pp. 37-38. See also Existence theorems for implicit functions of real variables, Bulletin of the American Mathematical Society, vol. 34 (1928), pp. 315-316. 
of several variables. A theorem that is an extension of that of Ettlinger, and follows as a result of Corollary 1 above, will now be stated.

TheOREM 2.2. Hypotheses: (a) $f(x, y)$ is summable on $X: a \leqq x \leqq b$ for each value of $y$ on $Y: Y_{1}<y<Y_{2}$; (b) the absolute continuity of $\int f(x, y) d x$ is uniform on $X$ for $y$ on $Y$; (c) for values of $x$ on $X_{0}, f(x, y)$ is a continuous function of $y$ on $Y$.

Conclusion: $F(x, y)=\int_{a}^{x} f(t, y) d t$ is continuous in $y$, uniformly with respect to $x$.

\section{MATRIX SOLUTIONS OF AN INFINITE DIFFERENTIAL SYSTEM}

According to the vector notation introduced in $\$ 2$ we may write system (1) as

$$
y^{\prime}(x)=A(x) y(x)
$$

Corresponding to this homogeneous system we have the non-homogeneous system

$$
y^{\prime}(x)=A(x) y(x)+b(x)
$$

where $b(x)$ is an arbitrary vector which is, together with its length, summable on the interval of definition $X: 0 \leqq x \leqq 1$. The differential system we may also write in the alternative integral form

$$
y(x)=\int_{0}^{x}[A(t) y(t)+b(t)] d t+y(0) .
$$

A solution of (3.2) is defined as an absolutely continuous vector whose length is bounded uniformly on $X$ by a finite constant and which satisfies the vector differential equation (3.2) on $X_{0}$.

With the condition on the matrix $A(x)$ that there exist a non-negative summable function $\phi(x)$ on $X$ such that for every vector $\xi$ of Hilbert space $|A(x) \xi| \leqq \phi(x)|\xi|$, it has been shown by Ettlinger* that there exists a unique solution of (3.2) satisfying the initial condition $y(0)=a$, where $a$ is an arbitrary vector in Hilbert space. Since $\phi(x)$ is of finite value on $X_{0}$, the the above condition demands that on $X_{0}$ the matrix $A(x)$ be limited by the corresponding finite value of $\phi(x)$. Since on $X-X_{0}$ the function $\phi(x)$ is infinite, we may say that $A(x)$ is limited by $\phi(x)$ on $X$. It then follows that for every vector $\xi$ of Hilbert space, $|\xi A(x)| \leqq \phi(x)|\xi| . \dagger$ Under this condition

* See second footnote on p. 286.

† H. T., p. 301. 
it follows from the existence theorem of Ettlinger that there exists a unique solution of the differential equation

$$
z^{\prime}(x)=-z(x) A(x)+b(x)
$$

satisfying the initial condition $z(0)=a$. The homogeneous equation

$$
z^{\prime}(x)=-z(x) A(x)
$$

is called the adjoint equation of (3.1)

Definition. Let $Y(x) \equiv\left(Y_{i j}(x)\right)$ be a matrix each column of which is a solution of the equation (3.1). Then $Y(x)$ is a matrix of (3.1). Similarly, if $Z(x)$ is a matrix each row of which is a solution of (3.4), then $Z(x)$ is a matrix of the adjoint equation. If for each value of $x$ on $X$ the matrices $Y(x)$ and $Z(x)$ are limited, then $Y(x)$ and $Z(x)$ are limited matrices of (3.1) and (3.4) respectively. In particular, $Y(x)$ is the principal matrix of (3.1) at a point $x_{0}$ of $X$ if $Y\left(x_{0}\right)=E$, the unit matrix. Likewise $Z(x)$ is the principal matrix of (3.4) at a point $x_{0}$ if $Z\left(x_{0}\right)=E$.

Ettlinger first showed the existence of a solution of the associated algebraic system and then that such a solution passes over in the limit to a solution of the differential system. We will here outline a proof of an existence theorem by the method of successive approximations where we do not consider the algebraic system, since the approximations there used are of use to us in considering the properties of the solution. The existence theorem we state as follows:

THEOREM 3.1. If $B(x)$ is a matrix each element of which is summable on $X$ and the matrix $\int_{0}^{x} B(t) d t$ is limited by a finite constant uniformly with respect to $x$ on $X$, and $C$ is a constant limited matrix, then there exists a unique absolutely continuous matrix $Y(x)$ such that

$$
Y(x)=\int_{0}^{x}[A(t) Y(t)+B(t)] d t+C,
$$

and $Y(x)$ is limited uniformly on $X$ by a finite constant.

Let

$$
\begin{aligned}
& Y^{(0)}(x)=\int_{0}^{x} B(t) d t+C, \\
& Y^{(k)}(x)=\int_{0}^{x}\left[A(t) Y^{(k-1)}(t)+B(t)\right] d t+C \quad(k=1,2, \cdots), \\
& H^{(0)}(x)=Y^{(0)}(x), \quad H^{(k)}(x)=Y^{(k)}(x)-Y^{(k-1)}(x) \quad(k=1,2, \cdots) .
\end{aligned}
$$

Then 
and

$$
Y^{(k)}(x)=\sum_{\alpha=0}^{k} H^{(\alpha)}(x),
$$

$$
H^{(k)}(x)=\int_{0}^{x} A(t) H^{(k-1)}(t) d t \quad(k=1,2, \cdots) .
$$

In the proof of Theorem 3.1 we make repeated use of the following lemma which we state without proof:

LEMMA 3.1. If $M(x)$ is a matrix each element of which is summable on $X$ and $M(x)$ is limited on $X$ by the summable function $\Phi(x)$, then for every $x$ on $X$ the matrix $\int_{0}^{x} M(t) d t$ is limited by the quantity $\int_{0}^{x} \Phi(t) d t .^{*}$

Since the matrix $\int_{0}^{x} B(t) d t$ is limited by a finite constant uniformly on $X$, there exists a finite constant $K$ which limits the matrix $\int_{0}^{x} B(t) d t+C$ uniformly on $X$. Now by (3.7),

$$
H^{(1)}(x)=\int_{0}^{x} A(t) H^{(0)}(t) d t,
$$

and since $A(x)$ is limited by $\phi(x)$ and $H^{(0)}(x)$ is limited by $K$, we have by Lemma 3.1 that $H^{(1)}(x)$ is limited by $K \int_{0}^{x} \phi(t) d t$. By mathematical induction we may prove that $H^{(k)}(x)$ is limited by the quantity

$$
K\left[\int_{0}^{x} \phi(t) d t\right]^{k} / k ! \quad(k=1,2, \cdots) .
$$

Therefore $\sum_{\alpha=0}^{\infty} H^{(\alpha)}(x)=\lim _{k \rightarrow \infty} Y^{(k)}(x)$ exists and is limited by the constant $K \exp \left[\int_{0}^{1} \phi(t) d t\right]$ uniformly on $X$. Let $Y(x)$ denote $\lim _{k \rightarrow \infty} Y^{(k)}(x)$. Then since $Y(x)-Y^{(k)}(x)=\sum_{\alpha=1}^{\infty} H^{(k+\alpha)}(x)$ is limited by the constant $\sum_{\alpha=1}^{\infty}\left[\int_{0}^{1} \phi(t) d t\right]^{k+\alpha}$ $[(k+\alpha) !]^{-1}$, the matrix $\int_{0}^{x} A(t)\left[Y(t)-Y^{(k)}(t)\right] d t$ is limited for every $x$ by the constant $\sum_{\alpha=1}^{\infty}\left[\int_{0}^{1} \phi(t) d t\right]^{k+1+\alpha} /(k+\alpha)$ ! and therefore

$$
\lim _{k \rightarrow \infty} \int_{0}^{x} A(t) Y^{(k)}(t) d t=\int_{0}^{x} A(t) Y(t) d t .
$$

Hence $Y(x)$ is a solution of (3.5).

* In particular let $M_{i 1}(x)=f_{i}(x), M_{i j}(x)=0$ if $j \neq 1$, where $f(x) \equiv\left(f_{i}(x)\right)$ is a vector which is, to gether with its length, summable on $X$. Then for every $x$ on $X, M(x)$ is limited by $|f(x)|$ and by the above lemma $\int_{0}^{x} M(t) d t$ is limited by $\int_{0}^{x}|f(t)| d t$ and therefore

$$
\left(\sum_{\alpha=1}^{\infty}\left[\int_{0}^{x} f_{\alpha}(t) d t\right]^{2}\right)^{1 / 2} \equiv\left|\int_{0}^{x} f(t) d t\right| \leqq \int_{0}^{x}|f(t)| d t .
$$

This theorem was used by Ettlinger in the proof of his existence theorem and is stated for the filrecase by Bliss, Annals of Mathematics, (2), vol. 6 (1904-1905), p. 59. This theorem will be usednite quently in this paper. 
Let $B_{k}(x)$ and $C_{k}$ denote the vectors $\left(B_{\alpha k}\right)$ and $\left(C_{\alpha k}\right)$ respectively and $Y_{k}^{*}(x) \equiv\left(Y_{\alpha k}^{*}(x)\right)$ be any solution of the vector differential equation

$$
y(x)=\int_{0}^{x}\left[A(t) y(t)+B_{k}(t)\right] d t+C_{k} .
$$

Then $Y^{* *}(x) \equiv\left(Y_{i j}{ }^{* *}(x)\right)$, where $Y_{i j}{ }^{* *}=Y_{i j}$ if $j \neq k$ and $Y_{i k}{ }^{* *}=Y_{i k^{*}}$, is a matrix that is a solution of (3.5) and is limited uniformly on $X$ by a finite constant $K^{*}$. Furthermore the matrix $Y^{(0)}(x)-Y^{* *}(x)$ is limited uniformly on $X$ by the constant $K^{*}+K$ and since

$$
Y^{(k)}(x)-Y^{* *}(x)=\int_{0}^{x} A(t)\left[Y^{(k-1)}(t)-Y^{* *}(t)\right] d t \quad(k=1,2, \cdots),
$$

it follows that for each integer $k$ the matrix $Y^{(k)}(x)-Y^{* *}(x)$ is limited by $\left[K^{*}+K\right]\left[\int_{0}^{x} \phi(t) d t\right]^{k} / k$ ! and therefore $Y^{* *}(x) \equiv Y(x)$ and the solution of (3.5) is unique.

COROLLARY 1. There exists a unique solution of (3.2) satisfying the initial condition $y(0)=a$, where $a$ is an arbitrary vector in Hilbert space.

COROLLARY 2. If $y(x)$ is a solution of (3.2), then $y(x)$ is a vector which is strongly continuous on $X$.

COROLlary 3. If $y(x)$ is a solution of (3.2), then $|y(x)|$ is an absolutely continuous function on $X$.

CoROllary 4. If $y(x)$ is a solution of (3.2), then the infinite series $\sum_{\alpha=1}^{\infty} y_{\alpha}^{2}(x)$ converges uniformly with respect to $x$ on $X$.

Corollary 5. If $Y(x)$ is a matrix of the equation (3.1) and for some point $x_{0}$ of $X$ the matrix $Y\left(x_{0}\right)$ is limited by a constant $l$, then $Y(x)$ is a limited matrix of (3.1) and is limited uniformly on $X$ by the constant $l \exp \left[\int_{0}^{1} \phi(t) d t\right]$.

If $U(x)$ and $V(x)$ are absolutely continuous matrices which are limited by a finite constant uniformly on $X$ and the matrices $d U / d x$ and $d V / d x$ are limited by a summable function on $X$, it follows from Corollary 2 of Theorem 2.1 that the matrix $U(x) V(x)$ is an absolutely continuous matrix and, on $X_{0}$,

$$
\frac{d(U V)}{d x}=\frac{d U}{d x} V+U \frac{d V}{d x}
$$

From this result we obtain

TheOREM 3.2. If $Y(x)$ and $Z(x)$ are limited matrices of (3.1) and (3.4) respectively, then the matrix $Z(x) Y(x)$ is a constant matrix on $X$. 
THEOREM 3.3. If $Y(x)$ is a limited matrix of (3.1) and for some point $x_{0}$ of $X$ the matrix $Y\left(x_{0}\right)$ possesses a unique limited reciprocal, then there exists a matrix $Z(x)$ which is the unique limited reciprocal of $Y(x)$ on $X$, and $Z(x)$ is $a$ limited matrix of the adjoint equation (3.4).

By hypothesis there exists a limited matrix $T$ such that $T Y\left(x_{0}\right)=Y\left(x_{0}\right) T$ $=E$. By Corollary 5 of Theorem 3.1 there exists a unique limited matrix $Z(x)$ of (3.4) such that $Z\left(x_{0}\right)=T$. Then $Z\left(x_{0}\right) Y\left(x_{0}\right)=E$ and by Theorem 3.2 we obtain $Z(x) Y(x)=E$ for every $x$ on $X$. If for some point $\bar{x}$ of $X$ the matrix $Y(x)$ possessed a left-hand reciprocal $V$ and $V \neq Z(\bar{x})$, by the same argument there would exist a limited matrix $Z^{*}(x)$ of (3.4) such that $Z^{*}(\bar{x})=V$ and therefore $Z^{*}(x) Y(x)=E$ for every $x$ on $X$. Since $Z^{*}\left(x_{0}\right)=Z\left(x_{0}\right)=T$ this is impossible as $Z(x)$ is the unique limited matrix of (3.4) satisfying the condition $Z\left(x_{0}\right)=T$. Hence $Z(x)$ is the unique left-hand reciprocal of $Y(x)$, and therefore it follows that $Z(x)$ is the unique limited reciprocal of $Y(x)$ on $X$.

CoRollaRY. If $Y(x)$ and $Z(x)$ are principal matrices of (3.1) and (3.2) respectively at some point $x=x_{0}$ of $X$, then $Y(x)$ and $Z(x)$ are unique limited reciprocals on $X$.

Definition. If $Y(x)$ is a limited matrix of (3.1) and possesses a unique limited reciprocal matrix on $X$, then $Y(x)$ is a matrix solution of (3.1). A matrix solution of the adjoint equation (3.4) is defined in a corresponding manner.

We will state without proof the following theorems:

TheOREM 3.4. If $Y(x)$ is a limited matrix of the equation (3.1) and $C$ is a constant limited matrix, then $Y(x) C$ is a limited matrix of (3.1).

COROLlARY. If $Y(x)$ is a matrix solution of (3.1) and $C$ is a constant limited matrix which possesses a unique limited reciprocal, then $Y(x) C$ is a matrix solution of (3.1).

THEOREM 3.5. If $Y(x)$ is a matrix solution of (3.1), then the most general limited matrix of (3.1) is of the form $Y(x) C$, where $C$ is a constant limited matrix.

COROLLARY. The most general matrix solution of the equation (3.1) is of the form $Y(x) C$, where $Y(x)$ is a matrix solution and $C$ is a constant limited matrix which has a unique limited reciprocal.

THEOREM 3.6. If $Y^{*}(x)$ is any matrix solution of (3.1) and the matrix $Z(x)$ is defined by the relation $Z(x) Y^{*}(x)=C$, where $C$ is a constant limited matrix, then $Z(x)$ is a limited matrix of (3.4).

† H. T., p. 312 
COROLlARY. If the constant limited matrix $C$ has a unique limited reciprocal, then $Z(x)$ is a matrix solution of the adjoint equation (3.4).

For the adjoint equation we have that the most general limited matrix of (3.4) is of the form $C Z(x)$, where $Z(x)$ is a matrix solution of (3.4) and $C$ is a constant limited matrix. Also every matrix solution of (3.4) may be written in the form $C Z(x)$, where $Z(x)$ is a matrix solution of (3.4) and $C$ is a constant limited matrix which has a unique limited reciprocal.

\section{INFINITE DIFFERENTIAL SYSTEMS INVOLVING A PARAMETER}

a. Continuity of the solution in $(x, \mu)$. We will now consider the vector differential equation

$$
y^{\prime}(x ; \mu)=A(x ; \mu) y(x ; \dot{\mu}),
$$

which is of the form (3.1) and in which each element of the matrix $A$ is a function of a parameter $\mu$. If for each value of $\mu$ on $L: L_{1}<\mu<L_{2}$ the matrix $A(x ; \mu)$ is limited by a summable function on $X: 0 \leqq x \leqq 1$, by Theorem 3.1 there exists for each value of $\mu$ on $L$ a unique solution of (4.1) satisfying the initial condition $y(0 ; \mu)=a(\mu)$, where $a(\mu)$ is for $\mu$ on $L$ a vector in Hilbert space.

THEOREM 4.1. Hypotheses: (a) There exists a summable function $\phi(x)$ such that $A(x ; \mu)$ is limited on $X$ by $\phi(x)$, uniformly with respect to $\mu$ on $L$; (b) for values of $x$ on $X_{0}$ the rows of $A(x ; \mu)$ are vectors which are strongly continuous on $L$; (c) $a_{i}(\mu)(i=1,2, \cdots)$ is continuous on $L$ and there exists $a$ finite constant $G$ such that $|a(\mu)|<G$ for every $\mu$ on $L$.

Conclusion: There exists a unique solution of (4.1) satisfying the initial condition $y(0 ; \mu)=a(\mu)$ and $y(x ; \mu)$ is continuous in $(x, \mu)$ on $X L$.

The following lemma will first be established:

LEMMA. If the matrix $A(x ; \mu)$ satisfies hypothesis (b) and $v(x ; \mu)$ is a vector each component of which is continuous on $L$ for values of $x$ on $X_{0}$ and there is a finite constant $K$ such that $|v(x ; \mu)|<K$ on $X L$, then $A(x ; \mu) v(x ; \mu)$ is a vector each of whose components is continumus in $\mu$ on $L$ for values of $x$ on $X_{0}$.

For every two points $\mu$ and $\mu^{\prime}$ of $L$ we have

$$
\begin{aligned}
& \left|\sum_{\alpha=n}^{\infty}\left[A_{i \alpha}\left(x ; \mu^{\prime}\right) v_{\alpha}\left(x ; \mu^{\prime}\right)-A_{i \alpha}(x ; \mu)\right] v_{\alpha}(x ; \mu)\right| \\
\leqq & \left|\sum_{\alpha=n}^{\infty}\left[A_{i \alpha}\left(x ; \mu^{\prime}\right)-A_{i \alpha}(x ; \mu)\right] v_{\alpha}(x ; \mu)\right| \\
+ & \left|\sum_{\alpha=n}^{\infty} A_{i \alpha}\left(x ; \mu^{\prime}\right)\left[v_{\alpha}\left(x ; \mu^{\prime}\right)-v_{\alpha}(x ; \mu)\right]\right|
\end{aligned}
$$


Now by the Lagrange-Cauchy inequality, $\uparrow$

$$
\begin{aligned}
& \left|\sum_{\alpha=n}^{\infty}\left[A_{i \alpha}\left(x ; \mu^{\prime}\right)-A_{i \alpha}(x ; \mu)\right] v_{\alpha}(x ; \mu)\right| \\
\leqq & {\left[\sum_{\alpha=n}^{\infty} v_{\alpha}^{2}(x ; \mu)\right]^{1 / 2}\left[\sum_{\alpha=n}^{\infty}\left[A_{i \alpha}\left(x ; \mu^{\prime}\right)-A_{i \alpha}(x ; \mu)\right]^{2}\right]^{1 / 2}, } \\
& \left|\sum_{\alpha=n}^{\infty} A_{i \alpha}\left(x ; \mu^{\prime}\right)\left[v_{\alpha}\left(x ; \mu^{\prime}\right)-v_{\alpha}(x ; \mu)\right]\right| \\
\leqq & {\left[\sum_{\alpha=n}^{\infty} A_{i \alpha}^{2}\left(x ; \mu^{\prime}\right)\right]^{1 / 2}\left[\sum_{\alpha=n}^{\infty}\left[v_{\alpha}\left(x ; \mu^{\prime}\right)-v_{\alpha}(x ; \mu)\right]^{2}\right]^{1 / 2} \cdot }
\end{aligned}
$$

Since each row of $A(x ; \mu)$ for $x$ on $X_{0}$ is strongly continuous on $L$ and since $|v(x ; \mu)|$ is bounded uniformly on $X L$, we have for values of $x$ on $X_{0}$ and for each value $\mu=\mu^{\prime}$ on $L$ that there exists for every positive $\epsilon$ a positive number $N_{\epsilon x_{\mu^{\prime} i}}$ and a positive quantity $\delta_{\varepsilon x \mu^{\prime} i}$ such that if $n>N_{\varepsilon x \mu^{\prime} i}$ and $\left|\mu-\mu^{\prime}\right|<\delta_{\varepsilon x \mu^{\prime} i}$ then

$$
\left|\sum_{\alpha=n}^{\infty} A_{i \alpha}\left(x ; \mu^{\prime}\right) v_{\alpha}\left(x ; \mu^{\prime}\right)-\sum_{\alpha=n}^{\infty} A_{i \alpha}(x ; \mu) v_{\alpha}(x ; \mu)\right|<\epsilon .
$$

Therefore, since for all values of $x$ on $X_{0}$ the finite sum $\sum_{\alpha=1}^{n} A_{i \alpha}(x ; \mu) v_{\alpha}(x ; \mu)$ is continuous in $\mu$, it follows that for values of $x$ on $X_{0}$ the infinite series $\sum_{a=1}^{\infty} A_{i \alpha}(x ; \mu) v_{\alpha}(x ; \mu)$ is a continuous function of $\mu$ on $L$.

We now apply the result of the lemma to the approximations $y^{(k)}(x ; \mu)$ which are defined by (3.6) and which converge to the solution $y(x ; \mu)$. We have

$$
\begin{aligned}
& y_{i}^{(0)}(x ; \mu)=a_{i}(\mu), \\
& y_{i}^{(k)}(x ; \mu)=a_{i}(\mu)+\int_{0}^{x} \sum_{\alpha=1}^{\infty} A_{i \alpha}(t ; \mu) y_{\alpha}{ }^{(k-1)}(t ; \mu) d t \quad(k=1,2, \cdots) .
\end{aligned}
$$

By hypothesis (c), $|a(\mu)|<G$ and therefore $y^{(k)}(x ; \mu)$ and $y(x ; \mu)$ are absolutely continuous on $X$, uniformly for $\mu$ on $L$, and $\left|y^{(k)}(x ; \mu)\right| \leqq G \exp \left[\int_{0}^{1} \phi(t) d t\right]$. Then if $y^{(k-1)}(x ; \mu)$ is for $x$ on $X_{0}$ a continuous function of $\mu$, it follows from the above lemma that $\sum_{\alpha=1}^{\infty} A_{i \alpha}(x ; \mu) y_{\alpha}{ }^{(k-1)}(x ; \mu)$ is for $x$ on $X_{0}$ a continuous function of $\mu$ on $L$. But $\left|\sum_{\alpha=1}^{\infty} A_{i \alpha}(x ; \mu) y_{\alpha}{ }^{(k-1)}(x ; \mu)\right|$ is bounded by the summable function $\phi(x) G \exp \left[\int_{0}^{1} \phi(t) d t\right]$ and since by hypothesis (c) each component of $a(\mu)$ is continuous on $L$, it follows from Theorem 2.2 that for fixed values of $x$ on $X$ each component of $y^{(1)}(x ; \mu)$ is a continuous function of $\mu$. By mathematical induction it can be shown that $y^{(k)}(x ; \mu)$ 
$(k=1,2, \cdots)$ is a continuous function of $\mu$. Since $|a(\mu)|$ is bounded uniformly on $L$, the sequence of vectors $y^{(k)}(x ; \mu)$ converges to the vector $y(x ; \mu)$ uniformly on $X L$ and therefore $y(x ; \mu)$ for each value of $x$ on $X$ is a continuous function of $\mu$. But the vector $y(x ; \mu)$ is absolutely continuous in $x$ on $X$, uniformly for $\mu$ on $L$, since $|a(\mu)|$ is bounded uniformly on $L$. Therefore $y(x ; \mu)$ is continuous in $(x, \mu)$ on $X L$. Also by the use of Corollary 1 of Theorem 2.1 we have that $y^{(k)}(x ; \mu)(k=1,2, \cdots)$ is continuous in $\mu$ uniformly with respect to $x$. Then since the sequence $y^{(k)}(x ; \mu)$ converges to $y(x ; \mu)$ uniformly on $X L$, we have that $y(x ; \mu)$ is continuous in $\mu$ uniformly for $x$ on $X$.

COROLlary. If for each value $\mu=\mu^{\prime}$ on $L$ there exists a positive number $d$ such that on $X L_{\mu^{\prime}}$, where $L_{\mu^{\prime}}: \mu^{\prime}-d \leqq \mu \leqq \mu^{\prime}+d$, the hypotheses of Theorem 4.1 are satisfied, then there exists a unique solution of (4.1) satisfying the initial condition $y(0 ; \mu)=a(\mu)$ and which is continuous in $(x, \mu)$ on $X L$.

Definition. An infinite matrix $M(x ; \mu)$ is a matrix of class $S$ on $X L$ if (a) $M(x ; \mu)$ is summable on $X$ for each value of $\mu$ on $L$ and is limited by a summable function $\theta(x)$ on $X$ uniformly for values of $\mu$ on $L$; (b) if $\mu$ and $\mu^{\prime}$ are any two points on $L$, then $M(x ; \mu)-M\left(x ; \mu^{\prime}\right)$ is limited by a function $C\left(x ; \mu ; \mu^{\prime}\right)$ which is summable on $X$ and such that $\lim _{\mu \rightarrow \mu^{\prime}} C\left(x ; \mu ; \mu^{\prime}\right)=0$ on $X_{0}$.

We now state the following auxiliary theorem:

THEOREM 4.2. If on the interval $x_{1} \leqq x \leqq x_{2},\left(x_{1}, x_{2}\right), u(x)$ is a non-negative continuous function and, for each value of $x$ on $\left(x_{1}, x_{2}\right)$,

$$
0 \leqq u(x) \leqq \int_{x_{1}}^{x}[\theta(t) u(t)+B(t)] d t+F(x),
$$

where $\theta(x)$ and $B(x)$ are non-negative summable functions on $\left(x_{1}, x_{2}\right)$ and $F(x)$ is a non-negative function bounded uniformly on $\left(x_{1}, x_{2}\right)$ by the constant $Q$, then for each value of $x$ on $\left(x_{1}, x_{2}\right)$,

$$
0 \leqq u(x) \leqq\left[\int_{x_{1}}^{x_{2}} B(t) d t+Q\right] \exp \left[\int_{x_{1}}^{x_{2}} \theta(t) d t\right] .
$$

THEOREM 4.3. If $A(x ; \mu)$ is a matrix of class $S$ on $X L$ and $Y(x ; \mu)$ is a matrix solution of the equation (4.1) which for some point $x=x_{0}$ of $X$ is a matrix of class $S$ on $X L$, then $Y(x ; \mu)$ is a matrix of class $S$ on $X L$.

Since $Y\left(x_{0} ; \mu\right)$ is constant with respect to $x$, if $Y\left(x_{0} ; \mu\right)$ is a matrix of class $S$ there exists a finite constant $K$ which limits $Y\left(x_{0} ; \mu\right)$ uniformly with respect to $\mu$ on $L$ and therefore $Y(x ; \mu)$ is limited uniformly on $X L$ by the 
constant $K^{*}=K \exp \left[\int_{0}^{1} \phi(t) d t\right]$. Also there is a finite quantity $\bar{C}\left(\mu ; \mu^{\prime}\right)$ which limits $Y\left(x_{0} ; \mu\right)-Y\left(x_{0} ; \mu^{\prime}\right)$ for each pair of points $\mu$ and $\mu^{\prime}$ on $L$ and $\lim _{\mu \rightarrow \mu^{\prime}}$ $\bar{C}\left(\mu ; \mu^{\prime}\right)=0$. We have

$$
\begin{aligned}
Y\left(x ; \mu^{\prime}\right)-Y(x ; \mu)= & \int_{x_{0}}^{x}\left[A\left(t ; \mu^{\prime}\right) Y\left(t ; \mu^{\prime}\right)-A(t ; \mu) Y(t ; \mu)\right] d t \\
& +Y\left(x_{0} ; \mu^{\prime}\right)-Y\left(x_{0} ; \mu\right)
\end{aligned}
$$

and for every vector $\xi$ of Hilbert space

$$
\begin{aligned}
& {\left[Y\left(x ; \mu^{\prime}\right)-Y(x ; \mu)\right] \xi=\int_{x_{0}}^{x}\left[A\left(t ; \mu^{\prime}\right)\left[Y\left(t ; \mu^{\prime}\right)-Y(t ; \mu)\right] \xi\right.} \\
& \left.+\left[A\left(t ; \mu^{\prime}\right)-A(t ; \mu)\right] Y(t ; \mu) \xi\right] d t+\left[Y\left(x_{0} ; \mu^{\prime}\right)-Y\left(x_{0} ; \mu\right)\right] \xi .
\end{aligned}
$$

Therefore

$$
\begin{aligned}
\left|\left[Y\left(x ; \mu^{\prime}\right)-Y(x ; \mu)\right] \xi\right| & \leqq \int_{x_{0}}^{x}\left[\phi(t)\left|\left[Y\left(t ; \mu^{\prime}\right)-Y(t ; \mu)\right] \xi\right|\right. \\
& \left.+C\left(t ; \mu ; \mu^{\prime}\right)|Y(t ; \mu) \xi|\right] d t+\left|\left[Y\left(x_{0} ; \mu^{\prime}\right)-Y\left(x_{0} ; \mu\right)\right] \xi\right|
\end{aligned}
$$

and by (4.6),

$$
\left|\left[Y\left(x ; \mu^{\prime}\right)-Y(x ; \mu)\right] \xi\right| \leqq\left[\int_{0}^{1} C\left(t ; \mu ; \mu^{\prime}\right) K^{*} d t+\bar{C}\left(\mu ; \mu^{\prime}\right)\right] \exp \left[\int_{0}^{1} \phi(t) d t\right]|\xi| .
$$

Hence the matrix $Y(x ; \mu)$ is a matrix of class $S$ on $X L$.

CoRollary 1. If $A(x ; \mu)$ is a matrix of class $S$ on $X L$ and $Y(x ; \mu)$ is the principal matrix of (4.1) at some point $x_{0}$ of $X$, then $Y(x ; \mu)$ is a matrix of class $S$ on $X L$.

Corollary 2. If in the differential equation (4.1) the matrix $A(x ; \mu)$ is a matrix of class $S$ on $X L$ and $a(\mu)$ is a vector which is strongly continuous on $L$, then the solution $y(x ; \mu)$ is for each value of $x$ a vector which is strongly continuous on $L$.

If $a(\mu)$ is strongly continuous, then $|a(\mu)|$ is bounded uniformly on every finite subinterval $L_{\mu^{\prime}}: \mu^{\prime}-d \leqq \mu \leqq \mu^{\prime}+d$ of $L$. Then $Y(0 ; \mu)$, where $Y_{i 1}(x ; \mu)=y_{i}(x ; \mu), Y_{i j}(x ; \mu)=0$ if $j>1$, is of class $S$ on $X L_{\mu^{\prime}}$ and the corollary follows directly.

An important example of an equation of the form (4.1) in which the matrix $A(x ; \mu)$ is a matrix of class $S$ is an equation in which the parameter enters linearly. Such an equation we may write as

$$
y^{\prime}(x ; \mu)=[A(x)+\mu B(x)] y(x ; \mu),
$$


where the matrices $A(x)$ and $B(x)$ are summable and limited by a summable function on $X$.

b. Differentiation of the solution of (4.1) with respect to the parameter $\mu$. Differentiation of the solution of a finite system of differential equations with respect to a parameter has been considered by Carathéodory. $\dagger$ For an infinite system of ordinary linear differential equations of the first order we have the following theorem.

ThEOREM 4.4. Hypotheses: (a) There exists a summable function $\phi(x)$ such that $A(x ; \mu)$ is limited on $X$ by $\phi(x)$, uniformly with respect to $\mu$ on $L$; (b) $a(\mu)$ is for each value of $\mu$ on $L$ a vector in Hilbert space each of whose components is continuous on $L$; (c) for $x$ on $X_{0}$ and at some point $\mu=\mu_{0}$ of $L$, $\partial A_{i j}(x ; \mu) / \partial \mu$ and $d a_{i}(\mu) / d \mu$ exist and $\left(d a_{i}\left(\mu_{0}\right) / d \mu\right)$ is a point in Hilbert space; (d) if $y\left(x ; \mu_{0}\right)$ is the solution for $\mu=\mu_{0}$ of $(4.1)$ such that $y\left(0 ; \mu_{0}\right)=a\left(\mu_{0}\right)$, then

$$
F_{i}\left(x ; \mu_{0}\right)=\sum_{\alpha=1}^{\infty} \frac{\partial}{\partial \mu} A_{i \alpha}\left(x ; \mu_{0}\right) y_{\alpha}\left(x ; \mu_{0}\right) \quad(i=1,2, \cdots)
$$

exists and $F\left(x ; \mu_{0}\right) \equiv\left(F_{i}\left(x ; \mu_{0}\right)\right)$ is a vector which is, together with its length, summable on $X$;

$$
\operatorname{LIM}_{\mu \rightarrow \mu_{0}} \frac{A(x ; \mu)-A\left(x ; \mu_{0}\right)}{\mu-\mu_{0}} y(x ; \mu)=\frac{\partial}{\partial \mu} A\left(x ; \mu_{0}\right) y\left(x ; \mu_{0}\right)
$$

for $x$ on $X_{0}$ and the absolute continuity of the integral

$$
\int_{0}^{x}\left|\frac{A(t ; \mu)-A\left(t ; \mu_{0}\right)}{\mu-\mu_{0}} y(t ; \mu)-\frac{\partial}{\partial \mu} A\left(t ; \mu_{0}\right) y\left(t ; \mu_{0}\right)\right| d t
$$

on $X$ is uniform with respect to $\mu$ on $L_{\mu_{0}}$, where $L_{\mu_{c}}: \mu_{0}-d \leqq \mu \leqq \mu_{0}+d$, and $d>0$;

$$
\underset{\mu \rightarrow \mu_{0}}{\operatorname{LIM}} \frac{a(\mu)-a\left(\mu_{0}\right)}{\mu-\mu_{0}}=\frac{d}{d \mu} a\left(\mu_{0}\right) .
$$

Conclusion: $\partial y(x ; \mu) / \partial \mu$ exists for $\mu=\mu_{0}$, and

(4.8) $\frac{\partial}{\partial \mu} y\left(x ; \mu_{0}\right)=\int_{0}^{x}\left[A\left(t ; \mu_{0}\right) \frac{\partial}{\partial \mu} y\left(t ; \mu_{0}\right)+\frac{\partial}{\partial \mu} A\left(t ; \mu_{0}\right) y\left(t ; \mu_{0}\right)\right] d t+\frac{d}{d \mu} a\left(\mu_{0}\right)$.

For every value of $\mu$ on $L_{\mu_{0}}$ we have

$$
\begin{aligned}
\frac{y(x ; \mu)-y\left(x ; \mu_{0}\right)}{\mu-\mu_{0}}= & \int_{0}^{x}\left[A\left(t ; \mu_{0}\right) \frac{y(t ; \mu)-y\left(t ; \mu_{0}\right)}{\mu-\mu_{0}}\right. \\
& \left.+\frac{A(t ; \mu)-A\left(t ; \mu_{0}\right)}{\mu-\mu_{0}} y(t ; \mu)\right] d t+\frac{a(\mu)-a\left(\mu_{0}\right)}{\mu-\mu_{0}} .
\end{aligned}
$$

† C. Carathéodory, Vorlesungen über reelle Funktionen, Berlin, 1918, p. 682. 
Now consider the equation

$$
u(x)=\int_{0}^{x}\left[A\left(t ; \mu_{c}\right) u(t)+F\left(t ; \mu_{0}\right)\right] d t+\frac{d}{d \mu} a\left(\mu_{0}\right),
$$

where $F\left(x ; \mu_{0}\right)$ is defined by hypothesis $(d)$. In view of the above hypotheses the equation (4.9) has a unique solution $u(x)$ and $|u(x)|$ is an absolutely continuous function on $X$. Then we have

$$
\begin{aligned}
\frac{y(x ; \mu)-y\left(x ; \mu_{0}\right)}{\mu-\mu_{0}}-u(x)= & \int_{0}^{x}\left\{A\left(t ; \mu_{0}\right)\left[\frac{y(t ; \mu)-y\left(t ; \mu_{0}\right)}{\mu-\mu_{0}}-u(t)\right]\right. \\
+ & \left.\frac{A(t ; \mu)-A\left(t ; \mu_{0}\right)}{\mu-\mu_{0}} y(t ; \mu)-\frac{\partial}{\partial \mu} A\left(t ; \mu_{0}\right) \dot{y}\left(t ; \mu_{0}\right)\right\} d t \\
& +\frac{a(\mu)-a\left(\mu_{0}\right)}{\mu-\mu_{0}}-\frac{d}{d \mu} a\left(\mu_{0}\right)
\end{aligned}
$$

and therefore

$$
\begin{aligned}
& \mid \begin{aligned}
\frac{y(x ; \mu)-y\left(x ; \mu_{0}\right)}{\mu-\mu_{0}}-u(x) \mid \leqq \int_{0}^{x}\left[\phi(t)\left|\frac{y(t ; \mu)-y\left(t ; \mu_{0}\right)}{\mu-\mu_{0}}-u(t)\right|\right. \\
\left.+B\left(t ; \mu ; \mu_{0}\right)\right] d t+C\left(\mu ; \mu_{0}\right),
\end{aligned}
\end{aligned}
$$

where

$$
B\left(t ; \mu ; \mu_{0}\right)=\left|\frac{A(t ; \mu)-A\left(t ; \mu_{0}\right)}{\mu-\mu_{0}} y(t ; \mu)-\frac{\partial}{\partial \mu} A\left(t ; \mu_{0}\right) y\left(t ; \mu_{0}\right)\right|
$$

and

$$
C\left(\mu ; \mu_{0}\right)=\left|\frac{a(\mu)-a\left(\mu_{0}\right)}{\mu-\mu_{0}}-\frac{d}{d \mu} a\left(\mu_{0}\right)\right| .
$$

Since, for each value of $\mu$,

$$
\left|\frac{y(x ; \mu)-y\left(x ; \mu_{0}\right)}{\mu-\mu_{0}}-u(x)\right|
$$

is continuous on $X$, by (4.6) we have

$$
\left|\frac{y(x ; \mu)-y\left(x ; \mu_{0}\right)}{\| \mu-\mu_{0}}-u(x)\right| \leqq\left[\int_{0}^{1} B\left(t ; \mu ; \mu_{0}\right) d t+C\left(\mu ; \mu_{0}\right)\right] \exp \left[\int_{0}^{1} \phi(t) d t\right] \text {. }
$$

In view of hypotheses (e) and (f) we have

$$
\operatorname{LIM}_{\mu \rightarrow \mu_{0}} \frac{y(x ; \mu)-y\left(x ; \mu_{0}\right)}{\mu-\mu_{0}}=u(x)
$$

and therefore $\partial y\left(x ; \mu_{0}\right) / \partial \mu$ is a point in Hilbert space for values of $x$ on $X$, and 


$$
\frac{\partial}{\partial \mu} y\left(x ; \mu_{0}\right)=\int_{0}^{x}\left[A\left(t ; \mu_{0}\right) \frac{\partial}{\partial \mu} y\left(t ; \mu_{0}\right)+\frac{\partial}{\partial \mu} A\left(t ; \mu_{0}\right) y\left(t ; \mu_{0}\right)\right] d t+\frac{d}{d \mu} a\left(\mu_{0}\right) .
$$

5. BOUNDARY CONDITIONS FOR AN INFINITE SYSTEM OF LINEAR DIFFERENTIAL EQUATIONS

a. Vector equations. We will state some theorems concerning the solution of vector equations in Hilbert space. The corresponding theorems for a finite system of linear equations involving a finite number of unknowns are well known. By a well known theorem $\dagger$ concerning infinite limited matrices, we have

THEOREM 5.1. A necessary and sufficient condition that a limited matrix $M$ possess a left-[right-]hand limited reciprocal is that there exist a positive quantity $K$ so that for all vectors $\xi$ of Hilbert space we have $K|M \xi| \geqq|\xi|[K|\xi M|$ $\geqq|\xi|]$.

Let $M \equiv\left(M_{i j}\right)$ and $N \equiv\left(N_{i j}\right)$ be two infinite limited matrices. Then we will denote by $(M ; N)$ the infinite matrix

$$
(M ; N)=\left\|\begin{array}{ccccc}
M_{11} & N_{11} & M_{12} & N_{12} & \cdots \\
M_{21} & N_{21} & M_{22} & N_{22} & \cdots \\
M_{31} & N_{31} & M_{32} & N_{32} & \ldots \\
\ldots & \ldots & \ldots & \ldots
\end{array}\right\| .
$$

Clearly $(M ; N)$ is a limited matrix. By $(M \mid N)$ we will denote the limited matrix

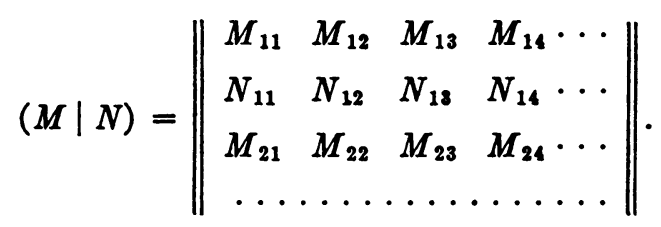

Definition. Two infinite matrices $M$ and $N$ belong to class $D_{1}\left[D_{2}\right]$ if they are limited matrices and there exists a matrix $\left(M^{\prime} ; N^{\prime}\right)\left[\left(M^{\prime} \mid N^{\prime}\right)\right]$ which has an infinite number of rows and columns and such that: (a) each column [row] of $\left(M^{\prime} ; N^{\prime}\right)\left[\left(M^{\prime} \mid N^{\prime}\right)\right]$ is a column [row] of $(M ; N)$. $[(M \mid N)] ;(\mathrm{b})\left(M^{\prime} ; N^{\prime}\right)\left[\left(M^{\prime} \mid N^{\prime}\right)\right]$ has a unique limited reciprocal; (c) there exist an infinite number of columns [rows] of $(M ; N)[(M \mid N)]$ which do not belong to $\left(M^{\prime} ; N^{\prime}\right)\left[\left(M^{\prime} \mid N^{\prime}\right)\right]$.

† This theorem follows from a more general theorem proved by Hyslop, $A$ contribution to the theory of bounded matrices and quadratic forms with an infinity of variables, Proceedings of the London Mathematical Society, vol. 24 (1925), pp. 264-304. See also Riesz, loc. cit., pp. 85, 86, et seq. 
The columns of $\left(M^{\prime} ; N^{\prime}\right)$ may be arranged in the sequence in which they appear in $(M ; N)$. That is, if the $i$ th column of $\left(M^{\prime} ; N^{\prime}\right)$ is the $s_{i}$ th column of $(M ; N)$, then $s_{1}<s_{2}<\cdots$. The infinite matrix whose columns are the columns of $(M ; N)$ which do not belong to $\left(M^{\prime} ; N^{\prime}\right)$ we will denote by $\left(M^{\prime \prime} ; N^{\prime \prime}\right)$. We will also consider the columns of $\left(M^{\prime \prime} ; N^{\prime \prime}\right)$ arranged in the sequence in which they appear in $(M ; N)$. Similarly, the rows of $\left(M^{\prime} \mid N^{\prime}\right)$ are arranged in the sequence in which they occur in $(M \mid N)$ and $\left(M^{\prime \prime} \mid N^{\prime \prime}\right)$ is the infinite limited matrix formed by arranging in their original order the rows of $(M \mid N)$ which do not belong to $\left(M^{\prime} \mid N^{\prime}\right)$. It is evident that in general the choice of the submatrices $\left(M^{\prime} ; N^{\prime}\right)$ and $\left(M^{\prime \prime} ; N^{\prime \prime}\right)$ or $\left(M^{\prime} \mid N^{\prime}\right)$ and $\left(M^{\prime \prime} \mid N^{\prime \prime}\right)$ is not unique.

Let $u \equiv\left(u_{\alpha}\right)$ be any vector in Hilbert space. Let $u^{\prime} \equiv\left(u_{\alpha}^{\prime}\right)$ be any vector such that $u_{\alpha}^{\prime}=u_{\sigma_{\alpha}}(a=1,2, \cdots), g_{1}<g_{2}<\cdots$, and such that there exist an infinite number of positive integers not belonging to the sequence $g_{1}, g_{2}, \ldots$. We will then write $u \equiv\left(u^{\prime} ; u^{\prime \prime}\right)$, where $u^{\prime \prime}$ is the vector whose $i$ th component is the $i$ th component of $u$ which does not belong to the sequence $u_{o_{1}}, u_{o_{2}}, \cdots$.

We will now prove the following lemma:

Lemma 5.1. If the matrices $M$ and $N$ are of class $D_{1}$, then the matrix $(M ; N)$ does not possess a left-hand reciprocal.

If $u$ is any vector, then the vectors $u^{\prime}$ and $u^{\prime \prime}$ can be so determined that $u \equiv\left(u^{\prime} ; u^{\prime \prime}\right)$ and

$$
(M ; N) u \equiv\left(M^{\prime} ; N^{\prime}\right) u^{\prime}+\left(M^{\prime \prime} ; N^{\prime \prime}\right) u^{\prime \prime},
$$

where the matrix $\left(M^{\prime} ; N^{\prime}\right)$ is an infinite matrix having a unique reciprocal and $\left(M^{\prime \prime} ; N^{\prime \prime}\right)$ is an infinite matrix. Since $\left(M^{\prime} ; N^{\prime}\right)$ has a unique reciprocal, for a given vector $u^{\prime \prime}$ the vector $u^{\prime}$ may be defined by $u^{\prime}=-\left(M^{\prime} ; N^{\prime}\right)^{-1}$ $\cdot\left(M^{\prime \prime} ; N^{\prime \prime}\right) u^{\prime \prime}$. Then $(M ; N) u=0$ and the vector $u$ is non-null if the vector $u^{\prime \prime}$ is non-null. Hence $(M ; N)$ does not have a left-hand reciprocal.

In a similar manner we have

Lemma 5.2. If the matrices $M$ and $N$ are of class $D_{2}$, then the matrix $(M \mid N)$ does not possess a right-hand reciprocal.

THEOREM 5.2. If $M$ and $N$ are matrices of class $D_{1}$, there exist matrices $B$ and $K$ which are of class $D_{2}$ and such that $(M ; N)(H \mid K)=0$.

We may write

$$
(M ; N)(H \mid K) \equiv\left(M^{\prime} ; N^{\prime}\right)\left(H^{\prime} \mid K^{\prime}\right)+\left(M^{\prime \prime} ; N^{\prime \prime}\right)\left(H^{\prime \prime} \mid K^{\prime \prime}\right),
$$

where $\left(M^{\prime} ; N^{\prime}\right)$ and $\left(M^{\prime \prime} ; N^{\prime \prime}\right)$ are infinite matrices and $\left(M^{\prime} ; N^{\prime}\right)$ possesses 
a unique limited reciprocal. Let $\left(H^{\prime \prime} \mid K^{\prime \prime}\right)$ be any limited matrix possessing a unique limited reciprocal. Then let

$$
\left(H^{\prime} \mid K^{\prime}\right)=-\left(M^{\prime} ; N^{\prime}\right)^{-1}\left(M^{\prime \prime} ; N^{\prime \prime}\right)\left(H^{\prime \prime} \mid K^{\prime \prime}\right) \text {. }
$$

Then we have $(M ; N)(H \mid K)=0$ and clearly the matrices $H$ and $K$ are of class $D_{2}$.

Definition. If $M$ and $N$ are matrices of class $D_{1}$ and $H$ and $K$ are matrices of class $D_{2}$ and such that

$$
\begin{aligned}
(M ; N)(H \mid K) & \equiv\left(M^{\prime} ; N^{\prime}\right)\left(H^{\prime} \mid K^{\prime}\right) \\
& +\left(M^{\prime \prime} ; N^{\prime \prime}\right)\left(H^{\prime \prime} \mid K^{\prime \prime}\right)=0,
\end{aligned}
$$

where the matrices $\left(M^{\prime} ; N^{\prime}\right),\left(M^{\prime \prime} ; N^{\prime \prime}\right),\left(H^{\prime} \mid K^{\prime}\right)$ and $\left(H^{\prime \prime} \mid K^{\prime \prime}\right)$ are infinite matrices and $\left(M^{\prime} ; N^{\prime}\right)$ and $\left(H^{\prime \prime} \mid K^{\prime \prime}\right)$ possess unique limited reciprocals, then the matrix $(H \mid K)$ will be said to be the complete matrix solution of the vector system

$$
(M ; N) u=0 .
$$

THEOREM 5.3. If $(H \mid K)$ is a complete matrix solution of the vector system (5.1), then every vector solution of this system is linearly dependent $\dagger$ on the columns of $(H \mid K)$.

If $v$ is any vector solution of the system (5.1) we have $(M ; N) v \equiv$ $\left(M^{\prime} ; N^{\prime}\right) v^{\prime}+\left(M^{\prime \prime} ; N^{\prime \prime}\right) v^{\prime \prime}=0$, where $v \equiv\left(v^{\prime} ; v^{\prime \prime}\right)$. Now we have

$$
v^{\prime \prime}=\left(B^{\prime \prime} \mid K^{\prime \prime}\right)\left(H^{\prime \prime} \mid K^{\prime \prime}\right)^{-1} v^{\prime \prime},
$$

and since each component of $v^{\prime}$ is a linear expression in the components of $v^{\prime \prime}$, it follows that $v^{\prime}=\left(H^{\prime} \mid K^{\prime}\right)\left(H^{\prime \prime} \mid K^{\prime \prime}\right)^{-1} v^{\prime \prime}$. Therefore $v=(H \mid K)$ $\cdot\left(H^{\prime \prime} \mid K^{\prime \prime}\right)^{-1} v^{\prime \prime}$ and $v$ is a vector which is linearly dependent on the columns of $(H \mid K)$.

Let $H, H^{*}, K$ and $K^{*}$ be four limited infinite matrices. We define the infinite matrix $\left(H ; K \mid H^{*} ; K^{*}\right)$ as follows:

$$
\begin{aligned}
\left(H ; K \mid H^{*} ; K^{*}\right) & =\left((H ; K) \mid\left(H^{*} ; K^{*}\right)\right) \\
& =\left(\left(H \mid H^{*}\right) ;\left(K \mid K^{*}\right)\right)
\end{aligned}
$$

or

$\dagger$ We will say that an infinite sequence of vectors $v^{(1)}, v^{(2)}, \cdots$ are linearly dependent if there exists a non-null vector $\xi$ of Hilbert space such that $\xi_{\alpha} v_{i}(\alpha)=0(j=1,2, \cdots)$. If $\xi_{r} \neq 0$ then $v^{(r)}$ is linearly dependent on the remaining vectors. If a set of vectors are not linearly dependent we will say that they are linearly independent. This is a more general notion of linear dependence for an infinite set of vectors than that used by Kowalewski. See Kowalewski, Einführung in die Determinantentheorie, Leipzig, 1909, p. 424. 


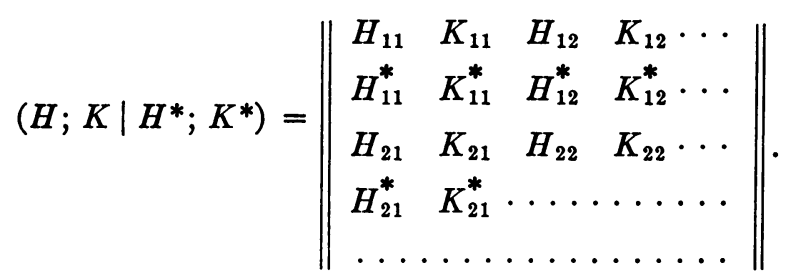

Suppose that the matrix $\left(H ; K \mid H^{*} ; K^{*}\right)$ has a unique limited reciprocal $G \equiv\left(G_{i j}\right)$. Then, considering $G\left(H ; K \mid H^{*} ; K^{*}\right)=E$, we have

$$
\begin{aligned}
G_{2 i-1,2 \alpha-1} H_{\alpha j}+G_{2 i-1,2 \alpha} H_{\alpha j}^{*} & =E_{i j}, \\
G_{2 i, 2 \alpha-1} H_{\alpha j}+G_{2 i, 2 \alpha} H_{\alpha j}^{*} & =0, \\
G_{2 i-1,2 \alpha-1} K_{\alpha j}+G_{2 i-1,2 \alpha} K_{\alpha j}^{*} & =0, \\
G_{2 i, 2 \alpha-1} K_{\alpha j}+G_{2 i, 2 \alpha} K_{\alpha j}^{*} & =E_{i j} \quad(i, j=1,2, \cdots) .
\end{aligned}
$$

Also if we consider the product $\left(H ; K \mid H^{*} ; K^{*}\right) G=E$, we have

$$
\begin{aligned}
H_{i \alpha} G_{2 \alpha-1,2 j-1}+K_{i \alpha} G_{2 \alpha, 2 j-1} & =E_{i j}, \\
H_{i \alpha} G_{2 \alpha-1,2 j}+K_{i \alpha} G_{2 \alpha, 2 j} & =0, \\
H_{i \alpha}^{*} G_{2 \alpha-1,2 j-1}+K_{i \alpha}^{*} G_{2 \alpha, 2 j-1} & =0, \\
H_{i \alpha}^{*} G_{2 \alpha-1,2 j}+K_{i \alpha}^{*} G_{2 \alpha, 2 j} & =E_{i j} \quad(i, j=1,2, \cdots) .
\end{aligned}
$$

The equations (5.2) and (5.3) give necessary and sufficient conditions that the matrix $G$ be the unique limited reciprocal of the matrix $\left(H ; K \mid H^{*} ; K^{*}\right)$. The conditions may be stated in matrix form as follows:

THEOREM 5.4. A necessary and sufficient condition that the limited matrix $\left(H ; K \mid H^{*} ; K^{*}\right)$ have a unique limited reciprocal is that there exist four limited matrices $R, S, R^{*}$ and $S^{*}$ satisfying the following matrix equations:

$$
\begin{aligned}
R^{*} H+R H^{*} & =E, \\
R^{*} K+R K^{*} & =0, \\
S^{*} H+S H^{*} & =0, \\
S^{*} K+S K^{*} & =E ; \\
H R^{*}+K S^{*} & =E, \\
H R+K S & =0, \\
H^{*} R^{*}+K^{*} S^{*} & =0, \\
H^{*} R+K^{*} S & =E .
\end{aligned}
$$

Furthermore, if $G \equiv\left(G_{i j}\right)$ is the unique limited reciprocal of $\left(H ; K \mid H^{*} ; K^{*}\right)$, then $G \equiv\left(R^{*} ; R \mid S^{*} ; S\right)$. 
Theorem 5.5. Let $M$ and $N$ be two matrices of class $D_{1}$ and let $\left(M^{\prime} ; N^{\prime}\right)$ denote an infinite matrix which has a unique limited reciprocal and each of whose columns is a column of $(M ; N)$; also, there exists an infinite matrix $\left(M^{\prime \prime} ; N^{\prime \prime}\right)$ whose columns are the columns of $(M ; N)$ which do not belong to $\left(M^{\prime} ; N^{\prime}\right)$. Let $T$ be a limited infinite matrix such that $T_{1}$, the infinite matrix whose columns are the columns of $T$ corresponding to the columns of $(M ; N)$ which belong to $\left(M^{\prime} ; N^{\prime}\right)$, is the zero matrix and $T_{2}$, the matrix whose columns are the columns of $T$ which do not belong to $T_{1}$, is a limited matrix which has a unique limited reciprocal. Then the limited matrix $\left(\left(M^{\prime} ; N^{\prime}\right) ;\left(M^{\prime \prime} ; N^{\prime \prime}\right) \mid T_{1} ; T_{2}\right)$ has a unique limited reciprocal.

It has been shown that a necessary and sufficient condition that $\left(\left(M^{\prime} ; N^{\prime}\right) ;\left(M^{\prime \prime} ; N^{\prime \prime}\right) \mid T_{1} ; T_{2}\right)$ have a unique limited reciprocal is that there exist a limited matrix which satisfies the equations (5.2) and (5.3). Define the matrix $G$ as follows:

$$
\begin{aligned}
\left(G_{2 i-1,2 j-1}\right) & \equiv\left(M^{\prime} ; N^{\prime}\right)^{-1} \\
\left(G_{2 i, 2 j-1}\right) & \equiv 0 \\
\left(G_{2 i, 2 j}\right) & \equiv T_{2}^{-1}, \\
\left(G_{2 i-1,2 j}\right) & \equiv-\left(M^{\prime} ; N^{\prime}\right)^{-1}\left(M^{\prime \prime} ; N^{\prime \prime}\right) T_{2}^{-1} .
\end{aligned}
$$

It readily follows that the matrix $G$ thus defined satisfies the equations (5.2) and (5.3) and is therefore the unique limited reciprocal of the matrix $\left(\left(M^{\prime} ; N^{\prime}\right) ;\left(M^{\prime \prime} ; N^{\prime \prime}\right) \mid T_{1} ; T_{2}\right)$.

We may now rearrange the columns of $\left(\left(M^{\prime} ; N^{\prime}\right) ;\left(M^{\prime \prime} ; N^{\prime \prime}\right) \mid T_{1} ; T_{2}\right)$ so that we have the matrix $\left(M ; N \mid M^{*} ; N^{*}\right)$, where $T \equiv\left(M^{*} ; N^{*}\right)$. Then $\left(M ; N \mid M^{*} ; N^{*}\right)$ also has a unique limited reciprocal which is given by the matrix formed by rearranging the rows of $\left(\left(M^{\prime} ; N^{\prime}\right) ;\left(M^{\prime \prime} ; N^{\prime \prime}\right) \mid T_{1} ; T_{2}\right)^{-1}$ in a corresponding manner.

THEOREM 5.6. If $M$ and $N$ are matrices of class $D_{1}$ and $(H \mid K)$ is a complete matrix solution of the vector system $(M ; N) u=0$, then there exist four limited matrices $M^{*}, N^{*}, H^{*}$ and $K^{*}$ such that the matrices $\left(M ; N \mid M^{*} ; N^{*}\right)$ and $\left(H^{*} ; H \mid K^{*} ; K\right)$ are unique limited reciprocals.

Since $M$ and $N$ are matrices of class $D_{1}$ we may write

$$
(M ; N)(H \mid K) \equiv\left(M^{\prime} ; N^{\prime}\right)\left(H^{\prime} \mid K^{\prime}\right)+\left(M^{\prime \prime} ; N^{\prime \prime}\right)\left(H^{\prime \prime} \mid K^{\prime \prime}\right)=0,
$$

where the matrices $\left(M^{\prime} ; N^{\prime}\right)$ and $\left(H^{\prime \prime} ; K^{\prime \prime}\right)$ have unique limited reciprocals. In Theorem 5.5 define $T_{2}$ as the matrix $\left(H^{\prime \prime} \mid K^{\prime \prime}\right)^{-1}$. Then in (5.4),

$$
\begin{aligned}
\left(G_{2 i-1,2 j}\right) & =\left(H^{\prime} \mid K^{\prime}\right), \\
\left(G_{2 i, 2 j}\right) & =\left(H^{\prime \prime} \mid K^{\prime \prime}\right) .
\end{aligned}
$$


Therefore by Theorem 5.5 the matrix $\left(M ; N \mid M^{*} ; N^{*}\right)$, where $\left(M^{*} ; N^{*}\right)=T$, has a unique limited reciprocal and $H^{*}$ and $K^{*}$ are uniquely determined so that $\left(H^{*} ; H \mid K^{*} ; K\right)$ is its reciprocal.

Clearly theorems analogous to Theorems $\mathbf{5 . 5}$ and 5.6 are true for matrices of class $D_{2}$.

b. Adjoint boundary conditions. Consider the infinite system of ordinary linear differential equations of the first order as given in $\$ 3$ by

$$
y^{\prime}(x)=A(x) y(x) .
$$

With this vector differential equation we associate boundary conditions

$$
M y(0)+N y(1)=0,
$$

where $M$ and $N$ are infinite matrices of class $D_{1}$. We have that the adjoint system of equations is

$$
z^{\prime}(x)=-z(x) A(x) .
$$

Consider now the vector equation

$$
z(0) P+z(1) Q=0,
$$

where the matrix $(-P \mid Q)$ is a complete matrix solution of the vector system $(M ; N) u=0$. From Theorem 5.6 and the equations $\left(5.2^{\prime}\right)$ we have that there exist four limited matrices $M^{*}, N^{*}, P^{*}$ and $Q^{*}$ such that

Let

$$
\begin{aligned}
-P^{*} M-P M^{*} & =E, \\
Q^{*} M+Q M^{*} & =0, \\
-P^{*} N-P N^{*} & =0, \\
Q^{*} N+Q N^{*} & =E .
\end{aligned}
$$

$$
\begin{aligned}
s_{i}(y) & =M_{i \alpha} y_{\alpha}(0)+N_{i \alpha} y_{\alpha}(1), \\
\bar{s}_{i}(y) & =M_{i \alpha}^{*} y_{\alpha}(0)+N_{i \alpha}^{*} y_{\alpha}(1), \\
t_{i}(z) & =P_{\alpha i} z_{\alpha}(0)+Q_{\alpha i} z_{\alpha}(1), \\
\bar{t}_{i}(z) & =P_{\alpha i}^{*} z_{\alpha}(0)+Q_{\alpha i z_{\alpha}}^{*}(1),
\end{aligned}
$$

Then in view of (5.5), (5.6) and (5.7) we have

$$
s_{\alpha}(y) \bar{t}_{\alpha}(z)+\bar{s}_{\alpha}(y) t_{\alpha}(z)=\left.y_{\alpha}(x) z_{\alpha}(x)\right|_{x=0} ^{x-1} .
$$

This equation is an identity for all vectors $y(x)$ and $z(x)$ which satisfy (5.5) and (5.6) respectively. We then say that the set of boundary conditions 
(5.6) is adjoint to the set (5.5) and that the differential system (3.4), (5.6) is adjoint to the differential system (3.1), (5.5). $\dagger$

The matrices $P$ and $Q$ have been so chosen that the matrix $(-P \mid Q)$ is a complete matrix solution of the system $(M ; N) u=0$. There are an infinite number of distinct complete matrix solutions of this system, but according to Theorem 5.3 every solution of this system is linearly dependent on any complete matrix solution. Hence two different choices of the complete matrix solution of $(M ; N) u=0$ give sets of equations $z(0) P+z(1) Q=0$ which are equivalent.

If the matrices $M$ and $N$ have unique limited reciprocals $M^{-1}$ and $N^{-1}$, then we may choose $P=M^{-1}$ and $Q=N^{-1}$. Then the adjoint boundary conditions become

$$
z(0) M^{-1}+z(1) N^{-1}=0 .
$$

\section{A BOUNDARY VALUE PROBLEM FOR AN INFINITE SYSTEM OF ORDINARY LINEAR DIFFERENTIAL EQUATIONS}

a. Completely continuous matrices. We now consider the bilinear form

$$
\xi K_{\eta} \equiv \sum_{i=1}^{\infty} \sum_{j=1}^{\infty} K_{i j} \xi_{i} \eta_{j}
$$

in which $\xi \equiv\left(\xi_{\alpha}\right)$ and $\eta \equiv\left(\eta_{\alpha}\right)$ are vectors in Hilbert space. For every positive integer $n$, let

$$
\xi K_{n} \eta=\sum_{i=1}^{n} \sum_{j=1}^{n} K_{i j} \xi_{i} \eta_{j} .
$$

According to Hilbert $\ddagger$ we have the following

Definition. A bilinear form $\xi K \eta$ is completely continuous if for every positive $\epsilon$ there exists a positive $N(\epsilon)$ such that if $\xi$ and $\eta$ are any pair of vectors of Hilbert space whose moduli do not exceed unity, then for all integers $m$ and $n$ such that $m, n>N(\epsilon)$,

$$
\left|\xi K_{n} \eta-\xi K_{m} \eta\right|<\epsilon \text {. }
$$

$\dagger$ This definition of adjoint boundary conditions is equivalent for a finite system to that given by Bounitzky. See Bounitzky, loc. cit., p. 73, and also Bliss, loc. cit., p. 564. We might also define a set of boundary conditions $z(0) H+z(1) K=0$, where $H$ and $K$ are limited matrices, as being adjoint to the set (5.5) if for every vector $z(x)$ satisfying this set and every vector $y(x)$ satisfying (5.5) we have

$$
\left.z_{\alpha}(x) y_{\alpha}(x)\right|_{x=0} ^{x-1}=0 \text {. }
$$

In a manner analogous to that used by Bounitzky to establish necessary conditions for the adjoint boundary conditions, it can be shown then that there exist matrices $P$ and $Q$ such that $(-P \mid Q)$ is a complete matrix solution of $(M ; N) u=0$ and $z(0) P+z(1) Q=0$. Hence these two definitions are equivalent.

$\ddagger$ Hilbert, loc. cit., p. 147 and p. 175 . 
We will say that a matrix $K \equiv\left(K_{i j}\right)$ is completely continuous if it is the matrix of a bilinear form that is completely continuous. If $K$ is a completely continuous matrix, then clearly $K$ is a limited matrix. In view of Theorem 5.1 and a theorem of Hilbert, $\dagger$ we may state

THEOREM 6.1. If $K$ is a completely continuous matrix, then a necessary and sufficient condition that the vector equation $(E+K) u=0$ have only the identically vanishing solution is that the matrix $(E+K)$ posses's a unique reciprocal.

Hilbert $\ddagger$ has also proved the following theorems:

TheOREM A. If $K$ is a completely continuous matrix and $H$ is a limited matrix, then $K H$ and $H K$ are completely continuous matrices.

COROLlaRy. If $K$ is a completely continuous matrix such that $(E+K)$ has a unique limited reciprocal $(E+K)^{-1}$, then $(E+K)^{-1}$ is of the form $\left(E+K^{*}\right)$, where $K^{*}$ is a completely continuous matrix.

THEOREM B. If $K$ is a completely continuous matrix, then the vector system $(E+K) u=0$ has at most a finite number of linearly independent solutions.

THEOREM C. The number of linearly independent solutions of the system $(E+K) u=0$ is the same as the number of linearly independent solutions of the system $u(E+K)=0$.

Let $M \equiv\left(M_{i j}\right)$ be an infinite limited matrix. If $s$ is a positive integer, we will say that the matrix $H \equiv\left(H_{i j}\right)$ is formed by omitting the sth column of $M$ if

$$
\begin{array}{ll}
H_{i j}=M_{i j} & (j<s ; i=1,2, \cdots), \\
H_{i j}=M_{i j+1} & (j \geqq s ; i=1,2, \cdots) .
\end{array}
$$

In a similar way we define a matrix formed by omitting a row of $M$. This idea may be extended readily to defining a matrix formed by omitting a finite or infinite number of rows and columns of $M$.

THEOREM 6.2. Let $K$ be a completely continuous matrix. If $(E+K) u=0$ has exactly r linearly independent solutions, $U_{1}, U_{2}, \cdots, U_{r}$, where $U_{k} \equiv\left(U_{\alpha k}\right)$, then no matrix with an infinite number of rows and columns and formed from $(E+K)$ by omitting $s$ columns, $s<r$, and by omitting a countable number of rows has a unique limited reciprocal.

Let $G$ denote the matrix formed by omitting from $(E+K)$ the columns of subscripts $g_{1}, g_{2}, \cdots, g_{s}$, and the rows of subscripts $l_{1}, l_{2}, \cdots$, where

$\dagger$ Hilbert, loc. cit., p. 165.

‡ Hilbert, loc. cit., p. 152, p. 165 et seq. 
there are an infinite number of positive integers which do not belong to the sequence $l_{1}, l_{2}, \cdots$. Since $s<r$ there exist constants $h_{1}, h_{2}, \cdots, h_{r}$ which are not all zero and such that

$$
\sum_{\alpha=1}^{r} h_{\alpha} U_{g_{\boldsymbol{i}} \alpha}=0 \quad(i=1,2, \ldots, s) .
$$

If the matrix $G$ has a unique limited reciprocal and $u$ is a vector solution of $(E+K) u=0$, then every component of $u$ is expressible linearly in terms of $u_{o_{1}}, u_{o_{2}}, \cdots, u_{o_{0}}$. But $\sum_{\alpha=1}^{r} h_{\alpha} U_{\alpha}$ is a vector solution of $(E+K) u=0$ and in view of (6.4) we then have $\sum_{\alpha=1}^{r} h_{\alpha} U_{\alpha}=0$. Since this is impossible if $U_{1}, U_{2}, \cdots, U_{r}$ are linearly independent, we have that the matrix $G$ cannot possess a unique limited reciprocal.

THEOREM 6.3. If the system $(E+K) u=0$, where $K$ is a completely continuous matrix, has exactly $r$ linearly independent solutions, then there exists a matrix formed by omitting $r$ rows and $r$ columns of $(E+K)$ which possesses a unique limited reciprocal.

Let $U_{1}, \cdots, U_{r}$, where $U_{k} \equiv\left(U_{\alpha k}\right)$, denote the $r$ linearly independent solutions of $(E+K) u=0$. Then there exists a matrix

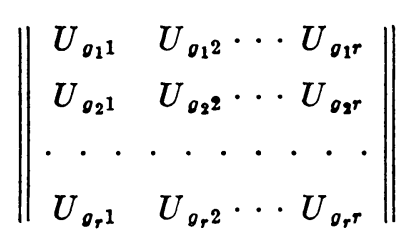

whose determinant is different from zero. By Theorem $\mathrm{C}$ there are exactly $r$ linearly independent solutions of the vector system $u(E+K)=0$. Let $V_{1}, V_{2}, \cdots, V_{r}$, where $V_{k} \equiv\left(V_{k \alpha}\right)$, denote' these solutions. Then there is a matrix

$$
\left\|\begin{array}{cc}
V_{1 q_{1}} & V_{1 q_{2}} \cdots V_{1 q_{r}} \\
V_{2 q_{1}} & V_{2 q_{3}} \cdots V_{2 q_{r}} \\
\cdot \cdot \cdot \cdot \cdot \cdot \cdot \cdot \\
V_{r q_{1}} & \cdots V_{r q_{r}}
\end{array}\right\|
$$

whose determinant is different from zero. Let $G$ denote the submatrix of $(E+K)$ formed by omitting the columns of subscripts $g_{1}, g_{2}, \cdots, g_{r}$ and the rows of subscripts $q_{1}, q_{2}, \cdots, q_{r}$. Suppose the matrix $G$ does not possess a unique limited reciprocal. $G$ is clearly of the form $(E+L)$, where $L$ is a completely continuous matrix. Furthermore, since the determinant $\left|\left(V_{i q_{j}}\right)\right|$ is different from zero, the rows of subscripts $q_{1}, q_{2}, \cdots, q_{r}$ are linearly dependent on the remaining rows of $(E+K)$. Then by Theorem 6.1 there 
would exist a non-null vector $v$ such that $G v=0$ and therefore there is a nonnull vector $u$ such that $u_{\sigma_{\alpha}}(\alpha=1,2, \cdots, r)$ is zero and $(E+K) u=0$. But this is impossible since $u$ must be by hypothesis linearly dependent on $U_{1}, U_{2}, \cdots, U_{r}$. Hence the matrix $G$ has a unique limited reciprocal.

b. A boundary value problem. We now consider a class of matrices which are functions of the variable $x$ on $X: 0 \leqq x \leqq 1$.

Definition. A matrix $H(x) \equiv\left(H_{i j}(x)\right)$ is a matrix of class $C^{*}$ if (a) $H_{i j}(x)(i, j=1,2, \cdots)$ is a Lebesgue summable function on $X$ and $H(x)$ is limited by a non-negative summable function $\phi(x)$ on $X$; (b) for values of of $x$ on $X_{0}$, the matrix $H(x)$ is a completely continuous matrix.

THEOREM 6.4. If $H(x)$ is a matrix of class $C^{*}$, then the matrix $\int_{0}^{z} H(t) d t$ is completely continuous uniformly on $X$.

For any $\epsilon>0$, let $Q_{n}(\epsilon)(n=1,2, \cdots)$ denote the set of all points $x$ on $X$ such that $\left|\xi H_{n}(x) \eta-\xi H(x) \eta\right|>\epsilon$ for some pair of vectors $\xi$ and $\eta$ whose moduli are not greater than unity. Since for values of $x$ on $X_{0}$ the matrix $H(x)$ is completely continuous, for every $\epsilon>0$ and every $\delta>0$ there exists a positive $N(\epsilon ; \delta)$ such that if $n>N(\epsilon ; \delta)$, then the interior measure of $Q_{n}(\epsilon)$ is less than $\delta . \dagger$ Therefore, since for every pair of vectors $\xi$ and $\eta$ of moduli not greater than unity the functions $\xi H_{n}(x) \eta$ and $\xi H(x) \eta$ are measurable on $X$ and $\left|\xi H_{n}(x) \eta\right| \leqq \phi(x)|\xi||\eta| \leqq \phi(x)$, it follows that for every $\epsilon>0$ there is an $N_{\epsilon}>0$ such that if $n, m>N_{\epsilon}$ then

$$
\left|\xi \int_{0}^{x} H_{n}(t) d t \eta-\xi \int_{0}^{x} H_{m}(t) d t \eta\right| \leqq \int_{0}^{x}\left|\xi H_{n}(t) \eta-\xi H_{m}(t) \eta\right| d t<\epsilon
$$

independent of the value of $x$ on $X$. Hence $\int_{0}^{x} H(t) d t$ is a completely continuous matrix uniformly on $X$.

Corollary. If $H(x)$ and $K(x)$ are matrices of class $C^{*}$ which are limited by the summable functions $\phi(x)$ and $\theta(x)$ respectively and $\phi(x) \theta(x)$ is a summable function, then $\int_{0}^{x} H(t) K(t) d t$ is a completely continuous matrix uniformly on $X$.

We now consider the infinite homogeneous differential vector equation

$$
y^{\prime}(x)=A(x) y(x),
$$

in which the matrix $A(x)$ is of class $C^{*}$ on $X: 0 \leqq x \leqq 1$. Such a system is a special form of the system (3.1) treated in $\$ 3$. Then we have

THEOREM 6.5. If $Y(x)$ is the principal matrix of (6.5) at the point $x=0$, then $Y(x)$ is of the form $E+R(x)$, where $R(x)$ is a matrix which is completely continuous uniformly on $X$.

† See de la Vallée Poussin, Cours d'Analyse, Paris, 1909, vol. 1, p. 252. 
With (6.5) we consider the boundary conditions

$$
\left(E+G_{1}\right) y(0)+\left(E+G_{2}\right) y(1)=0,
$$

where $G_{1}$ and $G_{2}$ are constant matrices which are completely continuous and $\left(E+G_{1}\right)$ and $\left(E+G_{2}\right)$ have unique limited reciprocals $\left(E+T_{1}\right)$ and $\left(E+T_{2}\right)$ respectively. That $T_{1}$ and $T_{2}$ are completely continuous follows from the corollary to Theorem A. The adjoint differential system is defined as

$$
\begin{gathered}
z^{\prime}(x)=-z(x) A(x), \\
z(0)\left(E+T_{1}\right)+z(1)\left(E+T_{2}\right)=0 .
\end{gathered}
$$

Clearly every finite system of ordinary linear differential equations of the first order in which the elements of the matrix $A(x)$ are summable on $X$ and the finite matrices $\left(E+G_{1}\right)$ and $\left(E+G_{2}\right)$ have non-vanishing determinants is a special case of the system considered.

Since the general solution of the equation (6.5) is of the form $Y(x) c$, where $Y(x)$ is the principal matrix at $x=0$ of (6.5) and $c$ is a constant vector in Hilbert space, if the system (6.5), (6.6) is compatible it is necessary and sufficient that there exist a non-null vector $c$ so that

$$
\left[\left(E+G_{1}\right) Y(0)+\left(E+G_{2}\right) Y(1)\right] c=0 .
$$

Since $\left[G_{1}+G_{2}+R(1)+G_{2} R(1)\right]$ is a completely continuous matrix, we have

THEOREM 6.6. A necessary and sufficient condition that (6.5), (6.6) be incompatible is that the matrix $(E+Q)$, where $Q=\left[G_{1}+G_{2}+R(1)+G_{2} R(1)\right] / 2$, have a unique limited reciprocal.

In view of Theorem $B$ we have

THEOREM 6.7. The system (6.5), (6.6) has at most a finite number of linearly independent solutions.

The corresponding non-homogeneous equation we write as

$$
\begin{gathered}
y^{\prime}(x)=A(x) y(x)+b(x), \\
\left(E+G_{1}\right) y(0)+\left(E+G_{2}\right) y(1)=h,
\end{gathered}
$$

where $A(x)$ is a matrix of class $C^{*}, b(x)$ is a vector which is, together with its length, summable on $X$, and $h \equiv\left(h_{\alpha}\right)$ is a constant vector in Hilbert space.

The following theorems are well known in the finite case and may be proved for the infinite system considered in a manner analogous to that used in the finite case. $\dagger$ 
Theorem 6.8. A necessary and sufficient condition that the non-homogeneous system (6.9), (6.10) have a unique solution is that (6.5), (6.6) be incompatible.

THEOREM 6.9. A necessary and sufficient condition that the adjoint system (6.7), (6.8) be compatible is that (6.5), (6.6) be compatible.

THEOREM 6.10. The number of linearly independent solutions of the adjoint system (6.7), (6.8) is the same as the number of linearly independent solutions of the system (6.5), (6.6).

We now consider a system of the form (6.5), (6.6) which involves a parameter $\mu$. Let $A(x ; \mu)$ be an infinite limited matrix which for all values of $\mu$ on $L: L_{1}<\mu<L_{2}$ is a matrix of class $C^{*}$ on $X: 0 \leqq x \leqq 1$; furthermore, let $A(x ; \mu)$ be a matrix of class $S$ on $X L$ as defined in $\$ 4$. We then have that $A(x ; \mu)$ is summable on $X$ and limited by a summable function $\phi(x)$ on $X$, uniformly for $\mu$ on $L$. Also $A(x ; \mu)-A\left(x ; \mu^{\prime}\right)$, for every pair of points $\mu$ and $\mu^{\prime}$ on $L$, is limited by a summable function $C\left(x ; \mu ; \mu^{\prime}\right)$ which is such that $\lim _{\mu \rightarrow \mu^{\prime}} C\left(x ; \mu ; \mu^{\prime}\right)=0$ on $X_{0}$. Let $G_{1}(\mu)$ and $G_{2}(\mu)$ be matrices which for each value of $\mu$ on $L$ are completely continuous and such that for every pair of points $\mu$ and $\mu^{\prime}$ on $L$ the matrices $G_{1}(\mu)-G_{1}\left(\mu^{\prime}\right)$ and $G_{2}(\mu)-G_{2}\left(\mu^{\prime}\right)$ are limited by finite quantities $C_{1}\left(\mu ; \mu^{\prime}\right)$ and $C_{2}\left(\mu ; \mu^{\prime}\right)$ respectively such that $\lim _{\mu \rightarrow \mu^{\prime}} C_{1}\left(\mu ; \mu^{\prime}\right)=0=\lim _{\mu \rightarrow \mu^{\prime}} C_{2}\left(\mu ; \mu^{\prime}\right)$. Furthermore $\left(E+G_{1}(\mu)\right)$ and $\left(E+G_{2}(\mu)\right)$ for each value of $\mu$ on $L$ possess unique limited reciprocals $\left(E+T_{1}(\mu)\right)$ and $\left(E+T_{2}(\mu)\right)$ respectively. We write this system as

$$
\begin{gathered}
y^{\prime}(x ; \mu)=A(x ; \mu) y(x ; \mu), \\
\left(E+G_{1}(\mu)\right) y(0 ; \mu)+\left(E \dot{+} G_{2}(\mu)\right) y(1 ; \mu)=0 .
\end{gathered}
$$

Clearly every finite system of ordinary linear differential equations of the first order in which the matrix $A(x ; \mu)$ is continuous in $\mu$ on $L$ for values of $x$ on $X_{0}$ and in which the matrices $E+G_{1}(\mu)$ and $E+G_{2}(\mu)$ are continuous in $\mu$ and have non-vanishing determinants is of the form (6.11), (6.12).

The system adjoint to (6.11), (6.12) we write

$$
\begin{gathered}
z^{\prime}(x ; \mu)=-z(x ; \mu) A(x ; \mu), \\
z(0 ; \mu)\left(E+T_{1}(\mu)\right)+z(1 ; \mu)\left(E+T_{2}(\mu)\right)=0 .
\end{gathered}
$$

In view of Theorem 6.10 we have

THEOREM 6.11. If $\mu=\mu^{\prime}$ is a characteristic number of the system (6.11), (6.12), its index, i.e., the number of linearly independent solutions corresponding to it, is finite and $\mu=\mu^{\prime}$ is also a characteristic number of the adjoint system (6.13), (6.14) of the same index.

For such a system we have 
Theorem 6.12. If for $\mu=\mu^{\prime}$ the system (6.11), (6.12) has r-fold compatibility, then there exists a positive number $\delta$ such that for all values of $\mu$ on $\left|\mu-\mu^{\prime}\right| \leqq \delta$ the system has at most r-fold compatibility.

Let $Y(x ; \mu)$ be the principal matrix of $(6.11)$ at $x=0$. By Corollary 1 of Theorem 4.3 the matrix $Y(x ; \mu)$ is of class $S$ on $X L$. Since $A(x ; \mu)$ is for each value of $\mu$ a matrix of class $C^{*}$, by Theorem 6.5 we have $Y(x ; \mu)=E+R(x ; \mu)$, where $R(x ; \mu)$ is completely continuous for values on $X L$. From Theorem 6.6 we have that a necessary and sufficient condition that the system (6.11), (6.12) be incompatible is that $E+Q(\mu)$, where $Q(\mu)=\left[G_{1}(\mu)+G_{2}(\mu)+R(1 ; \mu)\right.$ $\left.+G_{2}(\mu) R(1 ; \mu)\right] / 2$, have a unique limited reciprocal. Since $R(1 ; \mu)$ is of class $S$, the matrix $Q(\mu)-Q\left(\mu^{\prime}\right)$ is limited by a finite quantity $I\left(\mu ; \mu^{\prime}\right)$ for each pair of points $\mu$ and $\mu^{\prime}$ on $L$ and $\lim _{\mu \rightarrow \mu^{\prime}} I\left(\mu ; \mu^{\prime}\right)=0$. By hypothesis the system (6.11), (6.12) has $r$-fold compatibility for $\mu=\mu^{\prime}$. By Theorem 6.3 there exists then a submatrix $U(\mu)$ formed by omitting $r$ columns and $r$ rows of $E+Q(\mu)$ which has a unique limited reciprocal for $\mu=\mu^{\prime}$. Since $U(\mu)-U\left(\mu^{\prime}\right)$ is also limited by $I\left(\mu ; \mu^{\prime}\right)$ it follows in view of Theorem 5.1 that there exists a $\delta$ such that if $\left|\mu-\mu^{\prime}\right| \leqq \delta$, then $U(\mu)$ has a unique limited reciprocal. From Theorem 6.2 we then have that for $\mu$ on $\left|\mu-\mu^{\prime}\right| \leqq \delta$ the system (6.11), (6.12) has at most $r$ linearly independent solutions.

If we consider the case when $r=0$, we have

COROLLARY. The set of values of $\mu$ which are characteristic numbers of the system (6.11), (6.12) is a closed set of points on $L$.

ThEOREM 6.13. If $\mu^{\prime}$ is a point on $L$ and on the interval $\left|\mu-\mu^{\prime}\right| \leqq \delta$ the system (6.11), (6.12) has exactly $r$-fold compatibility, then there exists a solution $y(x ; \mu)$ of this system such that $\operatorname{LIM}_{\mu \rightarrow \mu^{\prime}} y(x ; \mu)=y\left(x ; \mu^{\prime}\right)$.

Let $U(\mu)$ be a matrix formed by omitting from $E+Q(\mu) r$ rows of subscripts $q_{1}, q_{2}, \cdots, q_{r}$ and $r$ columns of subscripts $g_{1}, g_{2}, \cdots, g_{r}$ and such that $U\left(\mu^{\prime}\right)$ has a unique limited reciprocal. It was shown in the proof of Theorem 6.12 that there exists an interval $\left|\mu-\mu^{\prime}\right| \leqq \delta^{*}\left(\delta^{*} \leqq \delta\right)$, on which $U(\mu)$ has a unique limited reciprocal. Let $D(\mu)$ denote the matrix formed by omitting the rows of subscripts $q_{1}, q_{2}, \cdots, q_{r}$ of $E+Q(\mu)$. Since by hypothesis the system (6.11), (6.12) has $r$-fold compatibility on $\left|\mu-\mu^{\prime}\right| \leqq \delta^{*}$, the rows of subscripts $q_{1}, q_{2}, \cdots, q_{r}$ are linearly dependent on the rows of $D(\mu)$. Since $D(\mu)-D\left(\mu^{\prime}\right)$ is limited by a quantity which converges to zero as $\mu$ approaches $\mu^{\prime}$, there exists a vector $c(\mu)$ such that $c(\mu)$ is non-null and $D(\mu) c(\mu)=0$ for $\mu$ on $\left|\mu-\mu^{\prime}\right| \leqq \delta^{*}$; furthermore, $c(\mu)$ is strongly continuous at $\mu=\mu^{\prime}$. Then $[E+Q(\mu)] c(\mu)=0$ on $\left|\mu-\mu^{\prime}\right| \leqq \delta^{*}$. Let $y(x ; \mu)=Y(x ; \mu) c(\mu)$, where $Y(x ; \mu)$ is the principal matrix of $(6.11),(6.12)$ at $x=0$. Since $Y(x ; \mu)$ is limited 
uniformly on $X L$ by a finite constant and since by Corollary 1 of Theorem 4.3 the matrix $Y(x ; \mu)$ is of class $S$, we have that $\operatorname{LIM}_{\mu-\mu^{\prime}} y(x ; \mu)=y\left(x ; \mu^{\prime}\right)$.

7. The Green's MATRIX FOR AN INFINITE SYSTEM OF DIFFERENTIAL EQUATIONS

Consider again the infinite differential system

$$
\begin{gathered}
y^{\prime}(x)=A(x) y(x), \\
\left(E+G_{1}\right) y(0)+\left(E+G_{2}\right) y(1)=0,
\end{gathered}
$$

where $A(x)$ is a summable matrix of class $C^{*}$ limited by the summable function $\phi(x)$ on $X: 0 \leqq x \leqq 1$, and $G_{1}$ and $G_{2}$ are constant infinite matrices which are completely continuous and $\left(E+G_{1}\right)$ and $\left(E+G_{2}\right)$ have unique limited reciprocals. The properties of this system have been considered in $\$ 6$.

A matrix $G(x ; t) \equiv\left(G_{i j}(x ; t)\right)$ which is defined on $0 \leqq x \leqq 1,0 \leqq t \leqq 1$, is said to be a Green's matrix of the system (7.1), (7.2) if it has the following properties:

(a) if $t$ is a point on $0<t<1$, then $G(x ; t)$ is a limited matrix, as defined in $\S 3$, of the equation (7.1) on $0 \leqq x<t$ and $t<x \leqq 1$;

$$
\begin{gathered}
G(t+0 ; t)-G(t-0 ; t)=E ; \\
\left(E+G_{1}\right) G(0 ; t)+\left(E+G_{2}\right) G(1 ; t)=0 .
\end{gathered}
$$

Suppose that the system (7.1), (7.2) is incompatible. By the same type of proof that is used to establish the existence of a Green's matrix for a finite system, we may show that a unique Green's matrix exists for the incompatible system (7.1), (7.2) and is of the form

$$
G(x ; t)=(1 / 2) Y(x)\left[\frac{|x-t|}{x-t} E+D W\right] Z(t), \dagger
$$

where $Y(x) Z(x)=E=Z(x) Y(x), D=[M Y(0)+N Y(1)]^{-1}$, and $W=M Y(0)-$ $N Y(1)$. In view of the theorems that are proved in $\S 3$, it is clear that the matrix $G(x ; t)$ is limited by a finite constant uniformly with respect to $x$ and $t$.

The system adjoint to (7.1), (7.2) is

$$
\begin{gathered}
z^{\prime}(x)=-z(x) A(x), \\
z(0)\left(E+T_{1}\right)+z(1)\left(E+T_{2}\right)=0,
\end{gathered}
$$

where $\left(E+T_{1}\right)$ and $\left(E+T_{2}\right)$ are the unique limited reciprocals of $\left(E+G_{1}\right.$ 
and $\left(E+G_{2}\right)$ respectively. We have shown in $\$ 6$ that $T_{1}$ and $T_{2}$ are matrices which are completely continuous.

As for a finite system we may prove $\dagger$

Theorem 7.1. If there exists a Green's matrix for the system (7.1), (7.2), then the adjoint system (7.5), (7.6) is incompatible.

THEOREM 7.2. The functions $H_{i k}(x ; t)=-G_{k i}(t ; x)$, where $G(x ; t)$ is the Green's matrix for the system (7.1), (7.2), are the elements of the Green's matrix for the adjoint system (7.5), (7.6) such that

(a) if $x$ is a point on $0<x<1$, then $G(x ; t)$ as a function of $t$ is a limited matrix of (7.5) on $0 \leqq t<x$ and $x<t \leqq 1$;

$$
\begin{gathered}
G(x ; x-0)-G(x ; x+0)=E \\
G(x ; 0)\left(E+T_{1}\right)+G(x ; 1)\left(E+T_{2}\right)=0 .
\end{gathered}
$$

Consider the non-homogeneous system

$$
y^{\prime}(x)=A(x) y(x)+b(x),
$$

where $A(x)$ is a matrix which is limited by a summable function $\phi(x)$ on $X$ and $b(x)$ is a vector which is, together with its length, summable on $X$. With this equation associate boundary conditions of the form (7.2).

Making use of the explicit form of $G(x ; t)$ we may prove, as for a finite system,

THEOREM 7.3. Every solution of the integral equation

$$
y(x)=\int_{0}^{1} G(x ; t) b(t) d t,
$$

where $G(x ; t)$ is defined by (7.4), is also a solution of the system (7.8), (7.2)and conversely every solution of the system (7.8), (7.2) can be expressed in the form (7.9).

We consider again the system

$$
\begin{gathered}
y^{\prime}(x ; \mu)=A(x ; \mu) y(x ; \mu), \\
\left(E+G_{1}(\mu)\right) y(0 ; \mu)+\left(E+G_{2}(\mu)\right) y(1 ; \mu)=0,
\end{gathered}
$$

where $A(x ; \mu)$ is a matrix of class $C^{*}$ and also a matrix of class $S$ on $X L$. For each value of $\mu$ on $L, G_{1}(\mu)$ and $G_{2}(\mu)$ are completely continuous matrices such that $E+G_{1}(\mu)$ and $E+G_{2}(\mu)$ have unique limited reciprocals $E+T_{1}(\mu)$ and $E+T_{2}(\mu)$ respectively; furthermore, for each pair of points $\mu$ and $\mu^{\prime}$

† Bounitzky, loc، cit., p. 77. 
on $L$ the matrices $G_{1}(\mu)-G_{1}\left(\mu^{\prime}\right)$ and $G_{2}(\mu)-G_{2}\left(\mu^{\prime}\right)$ are limited by finite quantities $C_{1}\left(\mu ; \mu^{\prime}\right)$ and $C_{2}\left(\mu ; \mu^{\prime}\right)$ respectively such that $\lim _{\mu \rightarrow \mu^{\prime}} C_{1}\left(\mu ; \mu^{\prime}\right)=0$ $=\lim _{\mu \rightarrow \mu^{\prime}} C_{2}\left(\mu ; \mu^{\prime}\right)$. Clearly for each value of $\mu$ for which the system (6.11), (6.12) is incompatible we may define uniquely a Green's matrix $G(x ; t ; \mu)$ in the same manner in which we defined a Green's matrix for the system (7.1), (7.2).

W. M. Whyburn $\dagger$ has considered the defining of a Green's matrix for a finite system of ordinary linear differential equations of the first order for values of the parameter which are characteristic numbers of the system. He showed how to construct a reduced system which is incompatible for the characteristic numbers of the original system and such that the coefficients of the reduced system differ by arbitrarily small amounts from the coefficients of the original system. The theorem as proved by Whyburn applies only to finite systems in which no component of the solution can be identically zero on $X$. We will here prove a related theorem for the infinite system (6.11), (6.12) which is more general in the finite case than Whyburn's theorem.

THEOREM 7.4. If for $\mu=\mu^{\prime}$ the system (6.11), (6.12) has $r$-fold compatibility, then there exists a reduced system (6.11'), (6.12') which is incompatible, where

$$
\begin{gathered}
y^{\prime}(x)=\left[A\left(x ; \mu^{\prime}\right)+P(x)\right] y(x), \\
\left(E+G_{1}\left(\mu^{\prime}\right)\right) y(0)+\left(E+G_{2}\left(\mu^{\prime}\right)\right) y(1)=0,
\end{gathered}
$$

and $P(x)$ is a matrix of non-negative continuous functions on $X$ which is limited by an arbitrarily assigned $\epsilon$ and at most $r$ of the elements of $P(x)$ are not identically zero on $X$.

In the proof of this theorem we will establish the following lemmas:

Lemma 1. If $y^{*}(x)$ is a solution of the system $\left(6.11^{\prime}\right),\left(6.12^{\prime}\right)$ and $z\left(x ; \mu^{\prime}\right)$ is a solution of the system (6.13), (6.14) for $\mu=\mu^{\prime}$, then

$$
\int_{0}^{1} z\left(x ; \mu^{\prime}\right) P(x) y^{*}(x) d x=0 .
$$

This follows immediately from the equation (5.9) since by hypothesis $y^{*}(x)$ and $z\left(x ; \mu^{\prime}\right)$ satisfy the boundary conditions $\left(6.12^{\prime}\right)$ and (6.14) respectively.

$\dagger$ W. M. Whyburn, On the Green's function for systems of differential equations, Annals of Mathematics, (2), vol. 28 (1927), pp. 291-300. 
LEMMA 2. If for $\mu=\mu^{\prime}$ the system (6.11), (6.12) has $r$-fold compatibility, then there exists a matrix $P(x)$ all of whose elements except one are identically zero and this element is a non-negative continuous function on $X$ whose absolute value is arbitrarily small and such that the system $\left(6.11^{\prime}\right),\left(6.12^{\prime}\right)$ has at most $(r-1)$-fold compatibility.

Since for $\mu=\mu^{\prime}$ the system (6.11), (6.12) has $r$-fold compatibility, there exists some component $y_{s}\left(x ; \mu^{\prime}\right)$ of a solution $y\left(x ; \mu^{\prime}\right)$ which is not identically zero on $X$. Let $x_{0}$ be a point of $X$ so that $y_{s}\left(x_{0} ; \mu^{\prime}\right) \neq 0$. Also since for $\mu=\mu^{\prime}$ the adjoint system (6.13), (6.14) has $r$-fold compatibility, there is some $k$ so that $z_{k}\left(x_{0} ; \mu^{\prime}\right) \neq 0$. Since $y_{s}\left(x ; \mu^{\prime}\right)$ and $z_{k}\left(x ; \mu^{\prime}\right)$ are continuous there is an interval $\left|x-x_{0}\right| \leqq d$ throughout which neither $y_{\bullet}\left(x ; \mu^{\prime}\right)$ nor $z_{k}\left(x ; \mu^{\prime}\right)$ vanishes. Now define a matrix $P^{*}(x)$ so that $P_{k s}{ }^{*}(x)$ is positive and continuous on $x_{0}-d<x<x_{0}+d$ and equal to zero elsewhere on $X$. We define $P_{i j}{ }^{*}(x), i \neq k$, $j \neq s$, as zero on $X$. Then

$$
\int_{0}^{1} z\left(x ; \mu^{\prime}\right) P^{*}(x) y\left(x ; \mu^{\prime}\right) d x=\int_{0}^{1} z_{k}\left(x ; \mu^{\prime}\right) P_{k s}^{*}(x) y_{s}\left(x ; \mu^{\prime}\right) d x \neq 0 .
$$

Now consider the system

$$
y^{\prime}\left(x ; \mu^{\prime} ; \theta\right)=\left[A\left(x ; \mu^{\prime}\right)+\theta P^{*}(x)\right] y\left(x ; \mu^{\prime} ; \theta\right),
$$

with the boundary conditions $\left(6.12^{\prime}\right)$. For $\theta=0$ the system (7.10), $\left(6.12^{\prime}\right)$ has $r$-fold compatibility. The matrix $A\left(x ; \mu^{\prime}\right)+\theta P^{*}(x)$, involving the parameter $\theta$, is a matrix of class $S$ for $x$ on $X$ and values of $\theta$ on $0 \leqq \theta \leqq 1$. Also, since by hypothesis the matrix $A\left(x ; \mu^{\prime}\right)$ is of class $C^{*}$ and since only one element of $P^{*}(x)$ is not identically zero, the matrix $A\left(x ; \mu^{\prime}\right)+\theta P^{*}(x)$ for each value of $\theta$ is of class $C^{*}$. Furthermore, since the boundary conditions do not involve $\theta$, the system (7.10), (6.12') is a system of the type (6.11), (6.12). Therefore there exists a positive $d$ such that for $0 \leqq \theta \leqq d$ the system (7.10), $\left(6.12^{\prime}\right)$ has at most $r$-fold compatibility. Suppose that for all values of the parameter $\theta$ on $0 \leqq \theta \leqq d$ the system $(7.10),\left(6.12^{\prime}\right)$ has exactly $r$-fold compatibility. Then as in Theorem (6.13) there would exist a solution $y\left(x ; \mu^{\prime} ; \theta\right), 0 \leqq \theta \leqq d$, and so that $\operatorname{LIM}_{\theta \rightarrow 0} y\left(x ; \mu^{\prime} ; \theta\right)=y\left(x ; \mu^{\prime} ; 0\right)$ and $y\left(x ; \mu^{\prime} ; 0\right)$ $=y\left(x ; \mu^{\prime}\right)$, the solution of $(6.11),(6.12)$ for $\mu=\mu^{\prime}$. From Lemma 1 we have that, for all values of $\theta$ on this interval,

$$
\int_{0}^{1} z\left(x ; \mu^{\prime}\right) P^{*}(x) y\left(x ; \mu^{\prime} ; \theta\right) d x=\int_{0}^{1} z_{k}\left(x ; \mu^{\prime}\right) P_{k s}^{*}(x) y_{s}\left(x ; \mu^{\prime} ; \theta\right) d x=0 .
$$

But this is impossible since 


$$
\lim _{\theta \rightarrow 0} \int_{0}^{1} z_{k}\left(x ; \mu^{\prime}\right) P_{k s}^{*}(x) y_{s}\left(x ; \mu^{\prime} ; \theta\right) d x=\int_{0}^{1} z_{k}\left(x ; \mu^{\prime}\right) P_{k s}^{*}(x) y_{s}\left(x ; \mu^{\prime}\right) d x \neq 0 .
$$

Therefore there exists a number $\theta_{1}, 0<\theta_{1} \leqq d$, such that the system (7.10), $\left(6.12^{\prime}\right)$ for $\theta=\theta_{1}$ has at most $(r-1)$-fold compatibility. Let $P(x)=\theta_{1} P^{*}(x)$. For this value of the matrix $P(x)$ the system $\left(6.11^{\prime}\right),\left(6.12^{\prime}\right)$ has at most $(r-1)$-fold compatibility.

If after choosing a reduced system as above described the reduced system is still compatible, we may choose a reduced system relative to the one already chosen and such that its degree of compatibility will be at least one lower than that of the previous system. Then after repeating this process at most $r$ times we obtain a reduced system which is incompatible. Clearly the elements of the matrix $P(x)$ can be chosen so that $P(x)$ is limited by an arbitrary number $\epsilon$, since we will need to make at most $r$ of the elements of $P(x)$ different from zero.

Now consider a differential system of the form (6.11), (6.12) which has only a countable number of characteristic numbers $\mu_{1}, \mu_{2}, \cdots$ on $L$ : $L_{1}<\mu<L_{2}$ and this set is such that $\mu_{1}<\mu_{2}<\mu_{3}<\cdots$ and the set has only one limit point, $L_{2}$. For every $\mu_{\alpha}$ we may define a matrix $P\left(x ; \mu_{\alpha}\right)$ satisfying the conditions of Theorem 7.4 and such that the corresponding reduced system of the form $\left(6.11^{\prime}\right),\left(6.12^{\prime}\right)$ is incompatible. A matrix $P(x ; \mu)$ may then be defined as follows:

$$
\begin{array}{lr}
P(x ; \mu)=P\left(x ; \mu_{\alpha}\right)+\frac{\mu-\mu_{\alpha}}{\mu_{\alpha+1}-\mu_{\alpha}}\left[P\left(x ; \mu_{\alpha+1}\right)-P\left(x ; \mu_{\alpha}\right)\right] & \text { on } \mu_{\alpha} \leqq \mu \leqq \mu_{\alpha+1}, \\
P(x ; \mu)=P\left(x ; \mu_{1}\right) & \text { on } L_{1}<\mu \leqq \mu_{1} .
\end{array}
$$

Then the system

$$
y^{\prime}(x ; \mu)=[A(x ; \mu)+P(x ; \mu)] y(x ; \mu),
$$

together with the boundary conditions (6.12), has no characteristic numbers in common with the system (6.11), (6.12). Then for each characteristic number $\mu_{\alpha}$ of the system (6.11), (6.12) we may define a Green's matrix for the reduced system (7.11), (6.12). Since the quantity $\epsilon$ by which the matrix $P(x ; \mu)$ is limited uniformly on $X L$ may be taken arbitrarily small, we will speak of the Green's matrix for the system (7.11), (6.12) for $\mu=\mu_{\alpha}$ $(\alpha=1,2, \cdots)$ as the Green's matrix for the characteristic numbers of the system (6.11), (6.12).

In order to define a Green's matrix for an infinite differential system which contains a parameter, it is not necessary that the system be of the form (6.11), (6.12). However, for a system of the form (6.11), (6.12) the conditions 
that $A(x ; \mu)$ be of class $S$ and that $G_{1}(\mu)-G_{1}\left(\mu^{\prime}\right)$ and $G_{2}(\mu)-G_{2}\left(\mu^{\prime}\right)$ be limited by quanitites that converge to zero as $\mu$ approaches $\mu^{\prime}$ are useful in considering the properties of the Green's matrix as a function of $\mu$.

Consider now the finite linear differential system

$$
y_{i}^{\prime}(x ; \mu)=\sum_{\alpha=1}^{n} A_{i \alpha}(x ; \mu) y_{\alpha}(x ; \mu) \quad(i=1,2, \cdots, n),
$$

with the boundary conditions

$$
\sum_{\alpha=1}^{n} M_{i \alpha}(\mu) y_{\alpha}(0 ; \mu)+\sum_{\alpha=1}^{n} N_{i \alpha}(\mu) y_{\alpha}(1 ; \mu)=0(i=1,2, \cdots, n),
$$

where we suppose that each element $A_{i j}(x ; \mu)$ is summable on $X$ and for values of $x$ on $X_{0}$ is a continuous function of $\mu$ on $L$. Furthermore, for each value of $\mu$ on $L$ the conditions of (7.13) are linearly independent and $M_{i j}(\mu)$ and $N_{i j}(\mu)(i, j=1,2, \cdots, n)$ are continuous functions on $L$. If the matrices $M(\mu) \equiv\left(M_{i j}(\mu)\right)$ and $N(\mu) \equiv\left(N_{i j}(\mu)\right)$ have non-vanishing determinants, then the finite system (7.12), (7.13) corresponds to the infinite system (6.11), (6.12) that we have considered. But in the more general case where the $n$ boundary conditions of (7.13) are merely linearly independent it is possible to prove for the system (7.12), (7.13) theorems that are analogous to Theorems 6.12 and 6.13. $\dagger$ For the system (7.12), (7.13) we can then prove a theorem which is analogous to Theorem 7.4.

† Whyburn proves these theorems for a finite differential system by a manner analogous to that used in the proof of Theorems 6.12 and 6.13.

UNIVERSITY OF TEXAS,

Austin, Texas 